KCL-PH-TH/2017-17, CERN-PH-TH/2017-071

UT-17-16, АCT-03-17, MI-TH-1752

UMN-TH-3623/17, FTPI-MINN-17/08

\title{
Starobinsky-like Inflation, Supercosmology and Neutrino Masses in No-Scale Flipped SU(5)
}

\author{
John Ellis ${ }^{a}$, Marcos A. G. Garcia ${ }^{b}$, Natsumi Nagata $^{c}$, \\ Dimitri V. Nanopoulos ${ }^{d}$ and Keith A. Olive ${ }^{e}$ \\ ${ }^{a}$ Theoretical Particle Physics and Cosmology Group, Department of Physics, \\ King's College London, London WC2R 2LS, United Kingdom; \\ Theoretical Physics Department, CERN, CH-1211 Geneva 23, Switzerland \\ ${ }^{b}$ Physics $\&$ Astronomy Department, Rice University, Houston, TX 77005, USA \\ ${ }^{c}$ Department of Physics, University of Tokyo, Bunkyo-ku, Tokyo 113-0033, Japan \\ ${ }^{d}$ George P. and Cynthia W. Mitchell Institute for Fundamental Physics and Astronomy, \\ Texas AEMM University, College Station, TX 77843, USA; \\ Astroparticle Physics Group, Houston Advanced Research Center (HARC), \\ Mitchell Campus, Woodlands, TX 77381, USA; \\ Academy of Athens, Division of Natural Sciences, Athens 10679, Greece \\ ${ }^{e}$ William I. Fine Theoretical Physics Institute, School of Physics and Astronomy, \\ University of Minnesota, Minneapolis, MN 55455, USA
}

\begin{abstract}
We embed a flipped SU(5) $\times$ U(1) GUT model in a no-scale supergravity framework, and discuss its predictions for cosmic microwave background observables, which are similar to those of the Starobinsky model of inflation. Measurements of the tilt in the spectrum of scalar perturbations in the cosmic microwave background, $n_{s}$, constrain significantly the model parameters. We also discuss the model's predictions for neutrino masses, and pay particular attention to the behaviours of scalar fields during and after inflation, reheating and the GUT phase transition. We argue in favor of strong reheating in order to avoid excessive entropy production which could dilute the generated baryon asymmetry.
\end{abstract}

April 2017 


\section{Introduction}

One of the biggest issues in particle physics is how to construct a testable theory of unification that goes beyond the Standard Model and also incorporates our phenomenological knowledge of neutrino masses and mixing. In parallel, one of the key issues in cosmology is how to construct a model of cosmological inflation that accommodates the current experimental constraints and relates it to particle physics in a testable way, e.g., by making specific predictions for reheating after inflation.

Flipped SU(5) $\times \mathrm{U}(1)$ [1-4] offers a promising framework for supersymmetric grand unification that offers resolutions of several important phenomenological issues in particle physics. For example, in addition to accommodating small neutrino masses [3, 5] 7], it provides a minimal mechanism for splitting the masses of the triplet and doublet components of the fiveplets of GUT Higgs fields [3]. Moreover, flipped $\mathrm{SU}(5) \times \mathrm{U}(1)$ can be extracted from string theory [4, 8 .

In parallel, a very attractive framework for constructing models of cosmological inflation [9 15] is provided by no-scale supergravity [16],17], which offers a positive semi-definite potential that accommodates naturally an asymptotically-flat direction that makes predictions similar to the Starobinsky model [18-20] that is highly consistent with the available cosmological data [21]. The next frontier in the phenomenology of Starobinsky-like models is to construct a model of post-inflationary reheating [15], which is testable in principle by a precise measurement of the tilt in the scalar perturbation spectrum, $n_{s}$. We addressed this issue recently in the framework of an $\mathrm{SO}(10)$ model of grand unification [22]: here we revisit Starobinsky-like inflation in the framework of the supersymmetric flipped $\mathrm{SU}(5) \times \mathrm{U}(1) \mathrm{GUT}$.

Working within such a specific framework enables - indeed, requires - us to address a wide range of related issues. For example, in connection with particle physics, one must check consistency with the available information on neutrino masses and mixing [3, 7, 23 26$]$, proton stability [27 30] and provide for the cold dark matter of the Universe [31 33]. Also, in connection with cosmology, one must check the evolution in the early Universe of the various scalar fields that necessarily appear in any more complete model of inflation [34]. Finally, one should aim at a successful scenario for generating the cosmological baryon asymmetry [35, 36].

In this paper we address these issues in the supersymmetric flipped $\mathrm{SU}(5) \times \mathrm{U}(1)$ GUT framework. We consider various possible identifications of the inflaton, and analyze the circumstances under which they can reproduce successful Starobinsky-like predictions for the tensor-to-scalar perturbation ratio, $r$, as well as the scalar tilt, $n_{s}$. We also study numerically the cosmological evolutions of the various GUT-singlet and massive sneutrino 
fields in the theory. Concerning reheating, we find a non-trivial link between the reheating temperature, and hence $n_{s}$, and the values of the neutrino masses. In the contexts of two specific scenarios for the masses and couplings of the singlet fields in the model, we show that experimental measurements of $n_{s}$ constrain significantly the model parameters. We also consider the GUT phase transition in the supersymmetric flipped $\mathrm{SU}(5) \times \mathrm{U}(1)$ model, following [6,37]. We argue that so long as the inflationary reheat temperature is sufficiently high (higher than the strong coupling scale associated with $\mathrm{SU}(5)$ ), excessive entropy release can be avoided, and it is relatively easy to obtain an adequate cosmological baryon asymmetry.

The layout of this paper is as follows. In Section 2 we introduce the flipped $\mathrm{SU}(5) \times$ U(1) GUT model that we study, Section 3 discusses cosmological inflation in this model, considering various possible inflaton assignments and the corresponding constraints on the model parameters that yield Starobinsky-like inflation. This Section also contains our numerical analysis of the behaviours of the various scalar fields during and after inflation. Neutrino masses and the decays of scalar fields are discussed in Section 4, and the GUT transition and scenarios for baryogenesis are discussed in Section 5. Finally, Section 6 summarizes and discusses our results.

\section{The No-Scale Flipped SU(5) $\times$ U(1) GUT model}

The field content of the flipped $\mathrm{SU}(5) \times \mathrm{U}(1)$ GUT we consider [1-4] consists of three generations of Standard Model (SM) matter fields, each with the addition of a right-handed neutrino, arranged in a $\mathbf{1 0}, \overline{\mathbf{5}}$, and $\mathbf{1}$ of $\mathrm{SU}(5)$ with the right-handed electrons and neutrinos, as well as the up- and down-type right-handed quarks, "flipped" with respect to a standard $\mathrm{SU}(5)$ assignment. The $\mathrm{SU}(5) \times \mathrm{U}(1)$ GUT group is subsequently broken to the $\mathrm{SM}$ group via $\mathbf{1 0}+\overline{\mathbf{1 0}}$ representations of $\mathrm{SU}(5)$, and subsequently to the $\mathrm{SU}(3) \times \mathrm{U}(1)$ symmetry via electroweak doublets in $\mathbf{5}+\overline{\mathbf{5}}$ representations. Our notations for the fields and their gauge representations are as follows:

$$
\begin{aligned}
& F_{i}=(\mathbf{1 0}, 1)_{i} \quad \ni\left\{d^{c}, Q, \nu^{c}\right\}_{i}, \\
& \bar{f}_{i}=(\overline{5},-3)_{i} \quad \ni\left\{u^{c}, L\right\}_{i} \text {, } \\
& \ell_{i}^{c}=(\mathbf{1}, 5)_{i} \quad \ni\left\{e^{c}\right\}_{i}, \\
& H=(\mathbf{1 0}, 1) \text {, } \\
& \bar{H}=(\overline{\mathbf{1 0}},-1) \text {, } \\
& h=(\mathbf{5},-2) \text {, } \\
& \bar{h}=(\overline{\mathbf{5}}, 2) \text {, }
\end{aligned}
$$


where the subscripts $i=1,2,3$ are generation indices that we suppress for clarity when they are unnecessary. Following the notation of [3], the states in $H$ will be labeled by the same symbols as in the $F_{i}$, but with an additional subscript: $d_{H}^{c}, Q_{H}, \ldots$, and states in $\bar{H}$ are similarly labelled including bars. States in $h$ are denoted by $\left(D, D, D, h^{-}, h^{0}\right)$ and in $\bar{h}$ by $\left(\bar{D}, \bar{D}, \bar{D}, h^{+}, \bar{h}^{0}\right)^{T}$. With these charge assignments, the hypercharge $Y$ is given by a linear combination of the $\mathrm{SU}(5)$ generator $T_{24} \equiv \operatorname{diag}(2,2,2,-3,-3) / \sqrt{60}$ and the $\mathrm{U}(1)$ charge $Q_{X}$ as

$$
Y=\frac{1}{\sqrt{15}} T_{24}+\frac{1}{5} Q_{X}
$$

Hence, the hypercharge $Y$ is not traceless in this model, contrary to a conventional $\mathrm{SU}(5)$ GUT.

The model also employs four singlet fields, which have no U(1) charges and are denoted by $\phi_{a}=(\mathbf{1}, 0), a=0, \ldots, 3$. As we comment below, it is also sufficient to consider only 3 singlets. In this case, the inflaton (associated with one combination of the singlets) participates in the neutrino mass matrix as in the $\mathrm{SO}(10)$ model discussed in Ref. [22].

The generic form for the superpotential of the theory can be written as 1

$$
\begin{aligned}
W= & \lambda_{1}^{i j} F_{i} F_{j} h+\lambda_{2}^{i j} F_{i} \bar{f}_{j} \bar{h}+\lambda_{3}^{i j} \bar{f}_{i} \ell_{j}^{c} h+\lambda_{4} H H h+\lambda_{5} \bar{H} \bar{H} \bar{h} \\
& +\lambda_{6}^{i a} F_{i} \bar{H} \phi_{a}+\lambda_{7}^{a} h \bar{h} \phi_{a}+\lambda_{8}^{a b c} \phi_{a} \phi_{b} \phi_{c}+\mu^{a b} \phi_{a} \phi_{b},
\end{aligned}
$$

where the indices $i, j$ run over the three fermion families, for simplicity we have suppressed gauge group tensor indices, and we impose a $\mathbb{Z}_{2}$ symmetry

$$
H \rightarrow-H
$$

that prevents the mixing of SM matter fields with Higgs colour triplets and members of the Higgs decuplets. This symmetry also suppresses the supersymmetric mass term for $H$ and $\bar{H}$, which has the advantage of suppressing the dangerous dimension-five proton decay operators as we discuss below. Expansion of the superpotential (3) in component fields reveals the following couplings

$$
W \supset \mu^{a b} \phi_{a} \phi_{b}+\lambda_{8}^{a b c} \phi_{a} \phi_{b} \phi_{c}+\lambda_{6}^{i a} \nu_{i}^{c} \nu_{\bar{H}}^{c} \phi_{a}
$$

for the SM gauge singlets $\phi_{a}$.

The Kähler potential for the model is assumed to have the no-scale form

$$
K=-3 \ln \left[T+\bar{T}-\frac{1}{3}\left(\left|\phi_{a}\right|^{2}+\left|\ell^{c}\right|^{2}+f^{\dagger} f+h^{\dagger} h+\bar{h}^{\dagger} \bar{h}+F^{\dagger} F+H^{\dagger} H+\bar{H}^{\dagger} \bar{H}\right)\right] .
$$

\footnotetext{
${ }^{1}$ Note that these couplings are exactly what would be allowed by $\mathrm{SO}(10)$. In the case where the $\mathrm{SU}(5) \times \mathrm{U}(1)$ gauge group is embedded into $\mathrm{SO}(10)$, the couplings $\lambda_{1}, \lambda_{2}$, and $\lambda_{3}$ are unified to a single Yukawa coupling. Moreover, in this case, additional chiral superfields need to be introduced so that $H$ and $\bar{H}$ are embedded into $\mathrm{SO}(10)$ representations.
} 
Therefore, in the absence of any moduli dependence of the gauge kinetic function, the scalar potential will have the form

$$
V=e^{2 K / 3}\left(\left|W_{i}\right|^{2}+\frac{1}{2} D^{a} D^{a}\right)
$$

where the $D$-term part of the potential in the limit of vanishing SM non-singlets has the form 2

$$
D^{a} D^{a}=\left(\frac{3}{10} g_{5}^{2}+\frac{1}{80} g_{X}^{2}\right)\left(\left|\tilde{\nu}_{i}^{c}\right|^{2}+\left|\tilde{\nu}_{H}^{c}\right|^{2}-\left|\tilde{\nu}_{\bar{H}}^{c}\right|^{2}\right)^{2}
$$

where we have rescaled the $\mathrm{U}(1)$ gauge coupling by a factor of $\sqrt{40}$; namely, the $\mathrm{U}(1)$ charges in (1) are expressed in units of $1 / \sqrt{40}$. The $\mathrm{SU}(5) \times \mathrm{U}(1)$ GUT symmetry is therefore broken along the $F$ - and $D$-flat direction $\left\langle\tilde{\nu}_{H}^{c}\right\rangle=\left\langle\tilde{\nu}_{\bar{H}}^{c}\right\rangle \neq 0$. These vevs, which can naturally be large thanks to the $F$ - and $D$-flatness, are induced by the soft supersymmetry-breaking masses. The resultant symmetry-breaking pattern is

$$
\mathrm{SU}(5) \times \mathrm{U}(1) \rightarrow \mathrm{SU}(3)_{C} \times \mathrm{SU}(2)_{L} \times \mathrm{U}(1)_{Y}
$$

Notice that this symmetry-breaking pattern is unique, contrary to the case of an ordinary supersymmetric SU(5) GUT, which has degenerate vacua, so that $\mathrm{SU}(5)$ may be broken into other gauge groups such as $\mathrm{SU}(4) \times \mathrm{U}(1)$. We also note that this model is free from any monopole problem, since the $\mathrm{SU}(5) \times \mathrm{U}(1)$ gauge group is not simple [2].

After $H$ and $\bar{H}$ develop vevs, thirteen gauge fields (out of the twenty-five in $\mathrm{SU}(5) \times$ $\mathrm{U}(1)$ ) acquire masses of order the GUT scale by absorbing the corresponding NambuGoldstone chiral superfields in $H$ and $\bar{H}$. The remaining seven chiral superfields in $H$ and $\bar{H}$ appear as physical states: one is a SM singlet and the others are the $d_{H}^{c}$ and $d_{\bar{H}}^{c}$. The former, which is a linear combination of $\nu_{H}^{c}$ and $\nu_{\bar{H}}^{c}$, is massless in the supersymmetric limit due to the presence of an $F$ - and $D$-flat direction in the potential, and has a mass of order the soft supersymmetry-breaking mass scale; we denote this combination by $\Phi$, and refer to it as the flaton. On the other hand, the $d_{H}^{c}$ and $d_{\bar{H}}^{c}$ are combined with the $D$ and $\bar{D}$ in $h$ and $\bar{h}$ via the couplings $\lambda_{4}$ and $\lambda_{5}$, respectively, have GUT-scale masses. The minimal supersymmetric Standard Model (MSSM) Higgs multiplets $h_{d}$ and $h_{u}$ in $h$ and $\bar{h}$, respectively, do not acquire masses through the vevs of $\nu_{H}^{c}$ and $\nu_{\bar{H}}^{c}$, and thus remain light. This realizes the so-called missing-partner mechanism [3, 38], which solves naturally the doublet-triplet splitting problem. We note that the flat direction is expected to be lifted by a higher order operator of the form $(H \bar{H})^{n} / M_{P}^{2 n-3}$ in the superpotential 3 . In order to obtain a GUT scale vev, we should have $n \geq 4$. As a result, we expect flaton and

\footnotetext{
${ }^{2}$ We can always rotate the vacuum expectation values (vevs) of $H$ and $\bar{H}$ into the $\tilde{\nu}_{H}^{c}$ and $\tilde{\nu}_{\bar{H}}^{c}$ directions, respectively, via $\mathrm{SU}(5)$ gauge transformations.

${ }^{3}$ We use natural units with $M_{P}^{-2}=8 \pi G_{N} \equiv 1$ throughout this paper.
} 
flatino masses to be of order $M_{\mathrm{GUT}}^{6} / M_{P}^{5}$, i.e., of order the supersymmetry-breaking scale, facilitating their decays into lighter MSSM particles.

In order to achieve successful electroweak symmetry breaking, we need a $\mu$-term for $h$ and $\bar{h}$ of order the supersymmetry-breaking scale. This can be generated via the Giudice-Masiero (GM) mechanism [39] or through the coupling $\lambda_{7}^{a}$ with supersymmetrybreaking scale vevs of $\phi_{a}$ [3], as in the next-to-minimal supersymmetric Standard Model (NMSSM). In order for $\phi_{a}$ to develop a TeV-scale vev, its supersymmetric mass term should be $\lesssim \mathcal{O}(1) \mathrm{TeV}$. In this case, a supersymmetry-breaking soft mass for $\phi_{a}$, which is driven negative by renormalization-group effects, allows $\phi_{a}$ to acquire a vev of order the soft mass scale, which can naturally explain the origin of the TeV-scale MSSM $\mu$-term. When only three singlets are included in the model, a $\mu$ term generated by the GM mechanism is necessary.

As we have mentioned above, a superpotential $\mu$-term for $H$ and $\bar{H}$ is suppressed by the $\mathbb{Z}_{2}$ symmetry, and thus the chirality flip between the color-triplet Higgs multiplets can occur only via the $\mu$-term for $h$ and $\bar{h} 4$ Since the dimension-five proton-decay process through the color-triplet Higgs exchange requires a chirality flip, this rate is suppressed by a factor of $\left(\mu / M_{H_{C}}\right)^{2}$, where $M_{H_{C}}$ denotes the color-triplet Higgs mass. As a consequence, this model can easily avoid the dimension-five proton decay limit from the $p \rightarrow K^{+} \bar{\nu}$ mode, $\tau\left(p \rightarrow K^{+} \bar{\nu}\right)>6.6 \times 10^{33}$ yrs [40], without relying on multi-TeV scale sfermions. This can enlarge the MSSM parameter space where both the correct dark matter density and the $125 \mathrm{GeV}$ Higgs boson mass are obtained [41].

In an $\mathrm{SU}(5) \times \mathrm{U}(1) \mathrm{GUT}$, the $\mathrm{SU}(3)_{C}$ and $\mathrm{SU}(2)_{L}$ gauge couplings unify at a high scale, $M_{32} \equiv M_{\mathrm{GUT}}$, into a single $\mathrm{SU}(5)$ gauge coupling $\alpha_{5}$ :

$$
\alpha_{3}\left(M_{32}\right)=\alpha_{2}\left(M_{32}\right)=\alpha_{5}\left(M_{32}\right)=0.0374
$$

where $M_{32}=1.2 \times 10^{16} \mathrm{GeV}$ when we use $\alpha_{3}\left(M_{Z}\right)=0.1181$ [42], the SM beta functions below $10 \mathrm{TeV}$, and the MSSM beta functions above $10 \mathrm{TeV}$ - both at two-loop level - and neglect threshold corrections. At this scale, the hypercharge gauge coupling $\alpha_{1} \equiv 5 \alpha_{Y} / 3$ is matched onto the $\mathrm{U}(1)$ gauge coupling $\alpha_{X}$ as

$$
\frac{25}{\alpha_{1}\left(M_{32}\right)}=\frac{1}{\alpha_{5}\left(M_{32}\right)}+\frac{24}{\alpha_{X}\left(M_{32}\right)} .
$$

\footnotetext{
${ }^{4}$ It is also possible that a GM term for $H$ and $\bar{H}$ could be generated in the Kähler potential through loop corrections accompanied by an explicit $\mathbb{Z}_{2}$-symmetry-breaking effect. Such a term would also contribute to the mass of the flaton and flatino. In addition, such an explicit $\mathbb{Z}_{2}$-symmetry-breaking term can prevent the generation of domain walls when the field $H$ acquires a vev. This $\mathbb{Z}_{2}$-symmetry-breaking effect would also generate a dimension-five proton-decay operator, but its contribution to the proton decay rate is suppressed by a factor of $\left(\mu_{H} / M_{H_{C}}\right)^{2}$ (where $\mu_{H}$ is the induced $\mu$-term for $H$ and $\bar{H}$ ), and thus does not lead to a proton decay problem.
} 
We see in these equations that the $\mathrm{U}(1)$ gauge coupling $\alpha_{X}$ is not necessarily equal to the $\mathrm{SU}(5)$ gauge coupling $\alpha_{5}$ at $M_{32}$. These couplings may unify at a higher scale, which is required if the $\mathrm{SU}(5) \times \mathrm{U}(1)$ gauge group is embedded into a simple group such as $\mathrm{SO}(10)$ at high energies, and in string constructions.

Since the unification of $\alpha_{3}$ and $\alpha_{2}$ should occur below the scale of complete unification, the first unification scale $M_{32}$ is expected to be smaller than the unification scale in the minimal SU(5) GUT [43, 44]. This indicates that the proton decay rate of the $p \rightarrow e^{+} \pi^{0}$ channel in this model, which is induced by the exchange of the $\mathrm{SU}(5)$ gauge bosons with masses around $M_{32}$, may be larger than that in the ordinary $\mathrm{SU}(5)$ GUT. The proton lifetime in the supersymmetric flipped $\mathrm{SU}(5) \times \mathrm{U}(1)$ model was evaluated in Ref. [29] as 5:

$$
\tau_{p}=4.6 \times 10^{35} \times\left(\frac{M_{32}}{10^{16} \mathrm{GeV}}\right)^{4} \times\left(\frac{0.0374}{\alpha_{5}\left(M_{32}\right)}\right)^{2} \mathrm{yrs} .
$$

This may be compared with the current experimental limit on the $p \rightarrow e^{+} \pi^{0}$ channel given by the Super-Kamiokande collaboration, which is $\tau\left(p \rightarrow e^{+} \pi^{0}\right)>1.6 \times 10^{34}$ yrs [46]. This sets a lower limit on the scale $M_{32}$ :

$$
M_{32}>4.3 \times 10^{15} \mathrm{GeV} \times\left(\frac{\alpha_{5}\left(M_{32}\right)}{0.0374}\right)^{\frac{1}{2}},
$$

which is generically satisfied when the low-energy matter content is the MSSM [43,44]. A part of the predicted range of proton lifetimes may be within the reach of future proton decay experiments, such as the Hyper-Kamiokande experiment [47].

\section{Inflation}

The inflaton, which we denote by $S$, is in general a linear combination of the singlet fields $\phi_{a}, a=0,1,2,36$. The asymptotically-flat Starobinsky potential is realized for $S$ if its superpotential takes the form 7 [9]

$$
W \supset m\left(\frac{S^{2}}{2}-\frac{S^{3}}{3 \sqrt{3}}\right),
$$

\footnotetext{
${ }^{5}$ The lifetime would be much shorter if there were additional vector-like multiplets at the TeV scale [30], since they would give a positive contribution to the gauge coupling beta functions, making the GUT-scale gauge coupling larger. In general, such extra matter multiplets at low energies can enhance proton decay rate considerably 45 .

${ }^{6}$ The discussion in this section is not affected by the number of singlets, and models with just three singlets with $a=0,1,2$ are also possible.

${ }^{7}$ An alternative choice of superpotential involving the moduli is $W=S(T-1 / 2)$ 48. Families of superpotentials that lead to the Starobinsky potential were discussed in [10].
} 
with $m \simeq 10^{-5}$ set by the measured primordial power spectrum amplitude. One would expect that the dimensionful couplings $\mu^{a b}$ in (5) would naturally be around the GUT scale $M_{\mathrm{GUT}} \sim 10^{-2}$, which is a few orders of magnitude above the intermediate scale set by the magnitude of $m$. Two scenarios for these couplings are possible: (1) the light state $S$ appears along with three heavy states upon diagonalizing a nearly-degenerate matrix $\mu^{a b}$, and (2) through an unspecified mechanism, some or all of the couplings are $\mu^{a b} \lesssim 10^{-5}$ at the inflation scale, implying that there may be more than one light scalar field, and in principle any of the $\phi_{a}$ could be identified with the inflaton. We consider both possibilities in what follows, and derive the corresponding phenomenological constraints on the model parameters.

\subsection{Scenario (1): hierarchy of singlet masses with one light state}

The simplest realization of (14) corresponds to the assumption that the state $S$ is the light eigenstate of a nearly-degenerate mass matrix $\mu^{a b}$. We identify $S$ with the rotated field $\phi_{0}^{D}$ in the diagonal basis denoted by subscripts $D$, where

$$
\mu_{D}^{a b}=\operatorname{diag}\left(m / 2, \mu_{D}^{11}, \mu_{D}^{22}, \mu_{D}^{33}\right), \quad \mu_{D}^{a b} \leq M_{\mathrm{GUT}},
$$

with $m \simeq 10^{-5}$. Such a light eigenstate exists if $\operatorname{det} \mu^{a b} \ll M_{\mathrm{GUT}}^{4}$.

In order to realize successful Starobinsky-like inflation as in (14), in the diagonal basis the Yukawa coupling must satisfy

$$
-3 \sqrt{3} \lambda_{8, D}^{000}=m
$$

For the remainder of this (sub)section we drop the index $D$, assuming implicitly that we refer to rotated fields and couplings.

We now investigate the conditions for sufficient inflation. Expanding the $(F$-term) scalar potential for the singlet fields reveals that large masses for the fields $\widetilde{\nu}^{c}$ and $\widetilde{\nu}_{\bar{H}}^{c}$ may be induced during inflation:

$$
\begin{aligned}
V_{F}= & e^{2 K / 3}\left[m^{2}\left|S-S^{2} / \sqrt{3}\right|^{2}+\sum_{i}\left(\left|\lambda_{6}^{i 0} \tilde{\nu}_{\bar{H}}^{c} S\right|^{2}+\left|\lambda_{6}^{i 0} \tilde{\nu}_{i}^{c} S\right|^{2}\right)+\cdots\right] \\
\simeq & \frac{3}{4} m^{2}\left(1-e^{-\sqrt{2 / 3} s}\right)^{2}+\frac{3}{4} \sinh ^{2}(\sqrt{2 / 3} s) \sum_{i}\left|\lambda_{6}^{i 0}\right|^{2}\left(\left|\tilde{\nu}_{\bar{H}}^{c}\right|^{2}+\left|\tilde{\nu}_{i}^{c}\right|^{2}\right) \\
& +\frac{1}{8} m^{2} e^{\sqrt{2 / 3} s}\left(\left|\tilde{\nu}_{\bar{H}}^{c}\right|^{2}+\sum_{i}\left|\tilde{\nu}_{i}^{c}\right|^{2}\right)+\cdots
\end{aligned}
$$

where $s=\sqrt{6} \tanh ^{-1}(S / \sqrt{3})$ denotes the canonically-normalized inflaton and the index $i=1,2,3$. In the second expression for $V_{F}$, we see the standard Starobinsky potential, 
followed by correction terms. At large $s$, we see the origin of the large masses for $\tilde{\nu}^{c}$ and $\tilde{\nu}_{\bar{H}}^{c}$. Therefore, for generic couplings, $\tilde{\nu}^{c}$ and the GUT-breaking field $\tilde{\nu}_{H}^{c}$ will be driven to zero in about one Hubble time during inflation, leaving the Universe in the symmetric phase at the end of inflation. A subsequent phase transition driven by the renormalization-group (RG) flow of the soft masses of $H$ and $\bar{H}$ via the couplings $\lambda_{4,5,6}$ can lead to the breaking of the $\mathrm{SU}(5) \times \mathrm{U}(1)$ symmetry [3] 8. No constraints on the couplings $\lambda_{6}^{i 0}$ are necessary for a successful inflationary phase, and we assume from now on that $\tilde{\nu}^{c}=\tilde{\nu}_{H}^{c}=0$ during inflation.

Another source for a deformation of the inflationary potential is the coupling of $S$ with the other $\phi_{i}$ fields. In order to determine its effect, we evaluate the gradient of the scalar potential during inflation:

$$
e^{-2 K / 3} \frac{\partial V}{\partial \bar{\phi}^{a}}=\sum_{b} W^{b}\left(\frac{2}{3} K_{a} \bar{W}_{b}+\bar{W}_{a b}\right) .
$$

During inflation, the fields $\phi_{j}$ (as well as any other scalars) get large masses,

$$
\frac{\partial^{2} V}{\partial \phi_{i} \partial \bar{\phi}^{j}}=\frac{2}{3} e^{K} m^{2}\left|S-S^{2} / \sqrt{3}\right|^{2} \delta_{j}^{i}+\cdots \simeq \frac{1}{8} m^{2} e^{\sqrt{2 / 3} s} \delta_{j}^{i}+\cdots \gg H^{2},
$$

and hence fluctuations displacing them from the origin can be neglected. Since all nonsinglet fields vanish, and we assume we are in the $\mu$-diagonal basis, the superpotential derivatives that appear in this expression correspond to

$$
\begin{aligned}
W^{i} & =3 \lambda_{8}^{00 i} S^{2}+2 \sum_{j}\left(\mu^{i j}+3 \lambda_{8}^{0 i j} S\right) \phi_{j}+3 \sum_{j, k} \lambda_{8}^{i j k} \phi_{j} \phi_{k} \\
W^{0} & =m\left(S-S^{2} / \sqrt{3}\right)+6 S \sum_{j} \lambda_{8}^{00 j} \phi_{j}+3 \sum_{j, k} \lambda_{8}^{0 j k} \phi_{j} \phi_{k}, \\
\bar{W}_{a b} & =2 \bar{\mu}_{a b}+6 \bar{\lambda}_{80 a b} \bar{S}+6 \sum_{j} \bar{\lambda}_{8 a b j} \bar{\phi}^{j} .
\end{aligned}
$$

We notice that, unless $\lambda_{8}^{00 i}=0$, (18) implies that the singlet fields will relax to nonzero values during inflation. The scenario in which $\phi_{i}=0$ is possible if $\mu^{a b}$ and $\lambda_{8}^{0 a b}$ can be diagonalized simultaneously. In that case, the couplings $\lambda_{8}^{00 i}$ are all absent and substituting $\phi_{i}=0$ into the effective potential yields

$$
V_{\mathrm{inf}}=\frac{3}{4} m^{2}\left(1-e^{-\sqrt{2 / 3} s}\right)^{2}
$$

i.e., simply the Starobinsky potential.

\footnotetext{
${ }^{8}$ We note that entropy could be released during this transition [6, 37, whose amount and potential danger we discuss in Section 5 .
} 
If $\mu^{a b}$ and $\lambda_{8}^{0 a b}$ are not simultaneously diagonalizable, then $\lambda_{8}^{00 i}$ may not vanish and thus $\phi_{i}$ may be non-zero during inflation. Since $m \simeq 10^{-5}$, and during inflation when $s$ is large, $S \sim \sqrt{3}$, we can disregard the contribution proportional to $W^{0}$ in (18) for the solution of the values of $\phi_{i}$, if in addition $\lambda_{8}^{00 i} \ll \lambda_{8}^{0 i j}$. This would imply that the instantaneous singlet vevs correspond approximately to the solutions of the equations $W^{i}=0$. The scalar potential during inflation then takes the form

$$
V \simeq e^{2 K / 3}\left|m\left(S-S^{2} / \sqrt{3}\right)+6 S \sum_{i} \lambda_{8}^{00 i} \phi_{i}+3 \sum_{j, k} \lambda_{8}^{0 j k} \phi_{j} \phi_{k}\right|^{2} .
$$

For $\lambda_{8}^{00 a} \ll \lambda_{8}^{0 i j}$, the singlet vevs are approximately given by the solution of the system of equations

$$
3 \lambda_{8}^{00 i} S^{2}+2 \sum_{j}\left(\mu^{i j}+3 \lambda_{8}^{0 i j} S\right) \phi_{j} \simeq 0
$$

and the effective potential (24) can be approximately written as

$$
\begin{aligned}
V_{\mathrm{inf}} & \simeq \frac{3}{4} m^{2}\left(1-e^{-\sqrt{2 / 3} s}\right)^{2} \\
& +\frac{3 \sqrt{3} m \sinh (\sqrt{2 / 3} s)}{2(1+\tanh (s / \sqrt{6}))}\left[2 \sqrt{3} \tanh (s / \sqrt{6}) \sum_{i} \lambda_{8}^{00 i} \phi_{i}+\sum_{i, j} \lambda_{8}^{0 i j} \phi_{i} \phi_{j}\right]+\text { h.c. }
\end{aligned}
$$

In general, the singlet vevs may be obtained by inverting the matrix $\left(\mu^{i j}+3 \lambda_{8}^{0 i j} S\right)$, but the resulting general expressions are not particularly enlightening. Instead, let us consider two limiting cases. First, let us assume that the couplings $\lambda_{8}^{0 i j} \gtrsim \mu^{i j}$. As $S=\mathcal{O}(1)$ during inflation, in this case we can disregard the $\mu^{a b}$ term in (25), and the effective potential (24) takes the approximate form

$$
\begin{aligned}
V_{\mathrm{inf}} & \simeq \frac{3}{4} m^{2}\left(1-e^{-\sqrt{2 / 3} s}\right)^{2}+\frac{27 m \sinh ^{2}(s / \sqrt{6})}{2(1+\tanh (s / \sqrt{6}))}\left(\sum_{i} \lambda_{8}^{00 i} \phi_{i}+\text { h.c. }\right) \\
& \simeq \frac{3}{4} m^{2}\left(1-e^{-\sqrt{2 / 3} s}\right)^{2}+\frac{27 \sqrt{3}}{4} m \Lambda e^{-s / \sqrt{6}} \sinh ^{3}(s / \sqrt{6}) .
\end{aligned}
$$

where we have defined

$$
\Lambda \equiv-\sum_{i, j}\left(\lambda_{8}^{0 i j}\right)^{-1} \lambda_{8}^{00 i} \lambda_{8}^{00 j}+\text { h.c. }
$$

The left panel of Fig. 1 shows the form of the scalar potential (28) as a function of $\Lambda$.

Starobinsky-like inflation with a total number of $e$-folds $N>60$ is realized only if $\Lambda \lesssim$ $10^{-10}$, which corresponds, schematically, to $\lambda_{8}^{00 i} \lesssim 10^{-5}\left(\lambda_{8}^{0 i j}\right)^{1 / 2} 9$. The left panel of Fig. 2

\footnotetext{
${ }^{9}$ In general, as we see also in later examples, Starobinsky-like inflation occurs if the deviation from the minimal Starobinsky potential is small for values of the inflaton field that are $\lesssim 6$.
} 

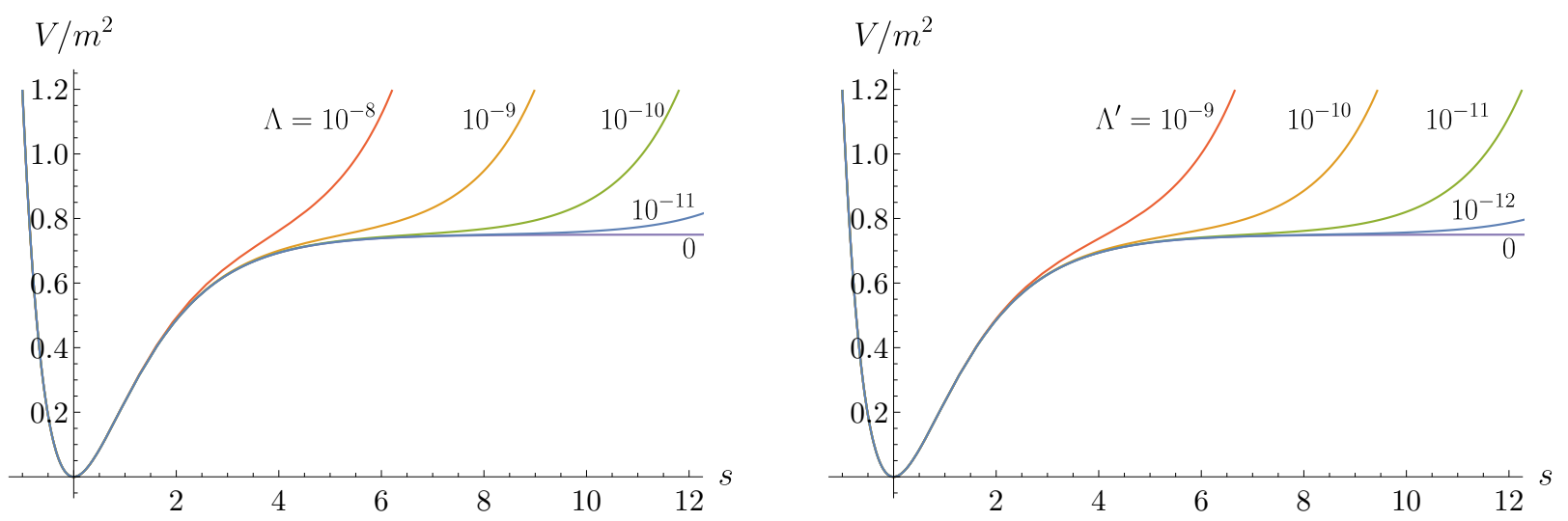

Figure 1: The effective inflationary potentials (28) and (30) for different values of $\Lambda$ (left) and $\Lambda^{\prime}$ (right), with $m=10^{-5}$. The curves labeled $\Lambda, \Lambda^{\prime}=0$ correspond to the Starobinsky potential (23).

shows the parametric dependence of the scalar tilt $n_{s}$ and the tensor-to-scalar ratio $r$ on $\Lambda$, and compares them to the $68 \%$ and 95\% CL constraints from Planck and other data [21]. We see that the model predicts $r \lesssim 0.007$ for the number of $e$-folds to the end of inflation, $N_{*}>$ 50 , within a factor $\sim 2$ of the Starobinsky prediction and far below the current experimental upper limit. On the other hand, for either $N_{*}=50$ or $N_{*}^{\max }$, Planck compatibility with the $95 \%$ CL range of $n_{s}$ is lost for $\lambda_{8}^{00 i} \gtrsim 10^{-4.8}\left(\lambda_{8}^{0 i j}\right)^{1 / 2}$, and for $N_{*}=60$ this occurs for $\lambda_{8}^{00 i} \gtrsim 10^{-4.9}\left(\lambda_{8}^{0 i j}\right)^{1 / 2}$. Here $N_{*}^{\max }$ is defined as the maximum number of e-folds after horizon crossing, which is compatible with the bound on the reheating temperature due to thermal production of gravitinos (see section 5.3 .3 for a detailed discussion). $N_{*}^{\max }$ is a function of the energy density at horizon crossing $V_{*}$, and therefore it is dependent on $\Lambda$; the curve shown in Fig. 2 takes into account this dependence, which is very weak, merely a $\lesssim 0.3 \%$ overall variation with respect to the Starobinsky limit $N_{*}^{\max } \simeq 53.3$.

If instead $\lambda_{8}^{0 i j} \lesssim \mu^{i j}$, as would be the case for a strongly-segregated inflaton sector, the effective potential can be approximated by

$$
V_{\text {inf }} \simeq \frac{3}{4} m^{2}\left(1-e^{-\sqrt{2 / 3} s}\right)^{2}+81 m \sinh ^{4}(s / \sqrt{6})(\tanh (s / \sqrt{6})-1) \sum_{i}\left[\mu_{i}^{-1}\left(\lambda_{8}^{00 i}\right)^{2}+\text { h.c. }\right],
$$

where $\mu^{a} \equiv \mu^{a a}$ since we have already assumed a basis where $\mu$ is diagonal. This potential is shown in the right panel of Fig. 1, where we have denoted

$$
\Lambda^{\prime}=-\sum_{a} \mu_{i}^{-1}\left(\lambda_{8}^{00 i}\right)^{2}+\text { h.c.. }
$$

In this case, Starobinsky-like inflation is obtained for $\Lambda^{\prime} \lesssim 10^{-11}$, or $\lambda_{8}^{00 i} \lesssim 10^{-5.5}\left(\mu^{i}\right)^{1 / 2} \sim$ $10^{-6.5}$. As shown in the right panel of Fig. 2, whereas the model prediction for $r$ is again 

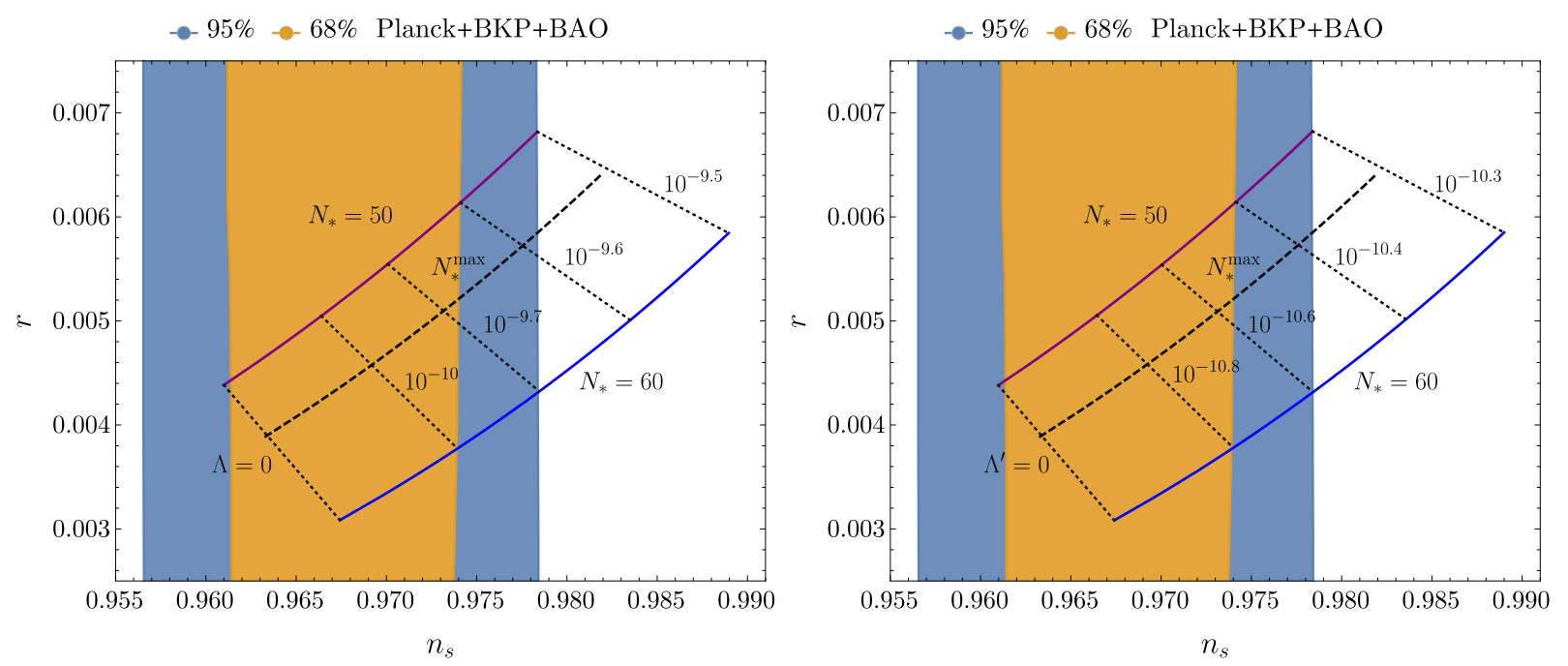

Figure 2: Parametric $\left(n_{s}, r\right)$ curves as functions of $\Lambda$ (left) and $\Lambda^{\prime}$ (right) for $N_{*}=50,60$ and $N_{*}^{\max } \simeq 53.3$, with the $68 \%$ and $95 \%$ CL constraints from Planck and other data [21] shown in the background. The solid curves show the parametric dependence using the analytical approximations (28) and (30). The dashed curve uses these analytical approximations together with (108) to determine the dependence at $N_{*}^{\max }$. The dotted curves are for illustrative values of $\Lambda, \Lambda^{\prime}$.

similar to the Starobinsky prediction, compatibility with the $95 \%$ Planck range of $n_{s}$ is lost for $\lambda_{8}^{00 i} \gtrsim 10^{-6.2}$ when $N_{*}=50$ or $N_{*}^{\max }$, and for $\lambda_{8}^{00 i} \gtrsim 10^{-6.3}$ when $N_{*}=60$.

We now discuss the dynamics of the scalar fields subsequent to inflation. If $\mu^{a b}$ and $\lambda_{8}^{0 a b}$ are simultaneously diagonalizable, the potential gradient for the non-inflaton singlet fields vanishes, implying they are not excited during reheating. If $\lambda_{8}^{0 a b}$ is not diagonal in the rotated basis, the singlets start oscillations about their minima, as they have non-zero vevs during inflation. Moreover, these oscillations are forced, driven by the oscillating inflaton $S$. To see this, let us investigate the potential gradient with respect to $\bar{\phi}^{i}$ :

$$
\begin{aligned}
\frac{\partial V}{\partial \bar{\phi}^{i}} \simeq \sum_{j} & {\left[4\left(\left|\mu^{i}\right|^{2} \delta_{i}^{j}+3 \bar{\mu}_{i} \lambda_{8}^{0 i j} S+3 \bar{\lambda}_{80 i j} \mu^{j} \bar{S}+9|S|^{2} \sum_{k} \lambda_{8}^{0 j k} \bar{\lambda}_{80 i k}\right) \phi_{j}+18 \bar{\lambda}_{80 i j} \lambda_{8}^{00 j}|S|^{2} S\right] } \\
+ & 6 \bar{\mu}_{i} \lambda_{8}^{00 i} S^{2}+\cdots .
\end{aligned}
$$

Since we assume that $\mu^{i} \gg m$ and $\left\langle\phi_{i}\right\rangle_{\text {inf }} \ll 1$, the fields $\phi_{i}$ will track quasi-statically the solution of the vev condition, $\partial V / \partial \bar{\phi}^{i}=0$. Naively, this implies that during reheating the singlets will remain small, $\phi_{i} \sim \lambda_{8}^{00 i}$. In general, however, if $\lambda_{8}^{0 i j} \gtrsim \mu^{i j}$, this may only be true during the first oscillation(s) of the inflaton $S$, as the approximation (32) breaks down 
if

$$
\operatorname{det}\left(\left|\mu^{i}\right|^{2} \delta_{i}^{j}+3 \bar{\mu}_{i} \lambda_{8}^{0 i j} S+3 \bar{\lambda}_{80 i j} \mu^{j} \bar{S}+9|S|^{2} \sum_{k} \lambda_{8}^{0 j k} \bar{\lambda}_{80 i k}\right)=0
$$

If a real solution exists, the $\phi_{i}$ exhibit resonant behaviour for $S \simeq 010$. Therefore, in this case, numerical integration of the equations of motion is necessary, as the singlets can in principle drain the energy density from the inflaton, and thereby be responsible for the eventual reheating of the Universe. We explore this effect in Section 3.3 ,

If no real solution for (33) exists, for $\lambda_{8}^{0 i j} \gtrsim \mu^{i j}$ the singlets evolve adiabatically with the inflaton oscillation. Modeling the inflaton oscillation as $s \simeq s_{0} \sin (m t) / m t$ and $H \simeq 2 / 3 t$, with $s_{0} \simeq 0.6$ [13], the time evolution of $\phi_{i}$ previous to the decay of $S$ can be approximated by

$$
\phi_{i}(t) \simeq-\frac{1}{2 \sqrt{2}} \sum_{j}\left(\lambda_{8}^{0 i j}\right)^{-1} \lambda_{8}^{00 j} s_{0}\left(\frac{\sin m t}{m t}\right), \quad t_{\mathrm{end}} \lesssim t \lesssim t_{\mathrm{reh}}
$$

disregarding corrections due to the finite mass of $\phi_{a}$ at the points for which $S=0$, which slightly overdamp the amplitude of the oscillations (see below). The timescales $t_{\text {end }}$ and $t_{\text {reh }}$ denote the times when inflation has ended and reheating has taken place respectively 11 . (34) implies that the ratio of the energy densities stored in the fields is given approximately by

$$
\frac{\rho_{\phi_{i}}}{\rho_{s}} \sim\left(\frac{\lambda_{8}^{00 i} s_{0}}{m}\right)^{2}\left(\frac{\sin m t}{m t}\right)^{2}
$$

Therefore, for $\lambda_{8}^{00 i} \lesssim 10^{-5}$, the energy density during reheating is always dominated by the oscillating inflaton, meaning that any phenomenological constraints related to the decay of the singlet fields, such as gravitino overproduction, can be reduced to the usual discussion for reheating bounds. Of course, this is the case as long as the singlet excitations decay sufficiently rapidly to avoid a matter-dominated era after reheating, which needs to be checked on a case-by-case basis (see Section 4.3). We further note that this limit on $\lambda_{8}^{00 i}$ is less constraining than the previous limit from sufficient inflation.

When the strong segregation condition $\lambda_{8}^{0 i j} \lesssim \mu^{i}$ is satisfied, the right-hand side of (32) is simply given by

$$
\frac{\partial V}{\partial \bar{\phi}^{i}} \simeq 4\left|\mu^{i}\right|^{2} \phi_{i}+6 \bar{\mu}_{i} \lambda_{8}^{00 i} S^{2}+18 \sum_{j} \bar{\lambda}_{80 i j} \lambda_{8}^{00 j}|S|^{2} S+\cdots,
$$

\footnotetext{
${ }^{10}$ Equation (33) is a polynomial equation of the form $\sum_{n=0}^{6} a_{n} S^{n}=0$, with $a_{0} \ll a_{1} \ll \cdots \ll a_{6}$, for which a solution (if it is real) is given by $S_{0} \simeq-a_{0} / a_{1} \ll 1$. As $S$ oscillates about the origin with an initial amplitude $S \sim \mathcal{O}(1)$, it crosses this point at least once.

${ }^{11}$ See [13] for more precise definitions of these quantities.
} 
and thus the evolution is always adiabatic. Under the same assumptions as in the previous case, the time evolution of $\phi_{i}$ previous to the decay of $S$ can be approximated by

$$
\phi_{i}(t) \simeq-\frac{3 \lambda_{8}^{00 i} s_{0}^{2}}{4 \mu^{i}}\left(\frac{\sin m t}{m t}\right)^{2}, \quad t_{\mathrm{end}} \lesssim t \lesssim t_{\mathrm{reh}}
$$

Substitution shows that the ratio of the energy densities is also given by (35). Thus, in this case reheating through $S$ decay also occurs, given that $\lambda_{8}^{00 i} \lesssim 10^{-5}$.

\subsection{Scenario (2): multiple light singlet states}

We now consider the case with multiple light singlet states, and assume that the relation

$$
-3 \sqrt{3} \lambda_{8}^{000}=2 \mu^{00}=m
$$

is realized off-diagonally, i.e., in a basis where $\phi_{0}$ is not a mass eigenstate. In this case, the superpotential parameters $\mu^{0 i}$ and $\lambda_{8}^{0 i j}$ must be constrained in order to allow for Starobinsky-like inflation. Despite the increased number of problematic parameters, the analysis is analogous to that in the previous Section. The singlet fields $\phi_{i}$ develop nonvanishing vevs during inflation, which can be found by solving (18), where in this case the superpotential derivatives are given by

$$
\begin{aligned}
& W^{i}=2 \mu^{0 i} S+3 \lambda_{8}^{00 i} S^{2}+2 \sum_{j}\left(\mu^{i j}+3 \lambda_{8}^{0 i j} S\right) \phi_{j}+3 \sum_{j k} \lambda_{8}^{i j k} \phi_{j} \phi_{k}, \\
& W^{0}=m\left(S-S^{2} / \sqrt{3}\right)+2 \sum_{j}\left(\mu^{0 j}+3 \lambda_{8}^{00 j} S\right) \phi_{j}+3 \sum_{j k} \lambda_{8}^{0 j k} \phi_{j} \phi_{k}, \\
& \bar{W}_{a b}=2 \bar{\mu}_{a b}+6 \bar{\lambda}_{80 a b} \bar{S}+6 \sum_{j} \bar{\lambda}_{8 a b j} \bar{\phi}^{j} .
\end{aligned}
$$

The effective potential during inflation then takes the form

$$
\begin{aligned}
V & =e^{2 K / 3}\left|m\left(S-S^{2} / \sqrt{3}\right)+2 \sum_{i}\left(\mu^{0 i}+3 \lambda_{8}^{00 i} S\right) \phi_{i}+3 \sum_{i, j} \lambda_{8}^{0 i j} \phi_{i} \phi_{j}\right|^{2} \\
\simeq & \frac{3}{4} m^{2}\left(1-e^{-\sqrt{2 / 3} s}\right)^{2} \\
& +\frac{\sqrt{3} m \sinh (\sqrt{2 / 3} s)}{2(1+\tanh (s / \sqrt{6}))}\left[2 \sum_{i}\left(\mu^{0 i}+3 \sqrt{3} \lambda_{8}^{00 i} \tanh (s / \sqrt{6})\right) \phi_{i}+3 \sum_{i, j} \lambda_{8}^{0 i j} \phi_{i} \phi_{j}\right]+\text { h.c. },
\end{aligned}
$$

where in the second line we have assumed that $\mu^{0 i}, \lambda_{8}^{00 i} \ll 1$, in which case the singlet vevs are approximately given by the solution of the system of equations

$$
2 \mu^{0 j} S+3 \lambda_{8}^{00 j} S^{2}+2 \sum_{k}\left(\mu^{j k}+3 \lambda_{8}^{0 j k} S\right) \phi_{k} \simeq 0 .
$$




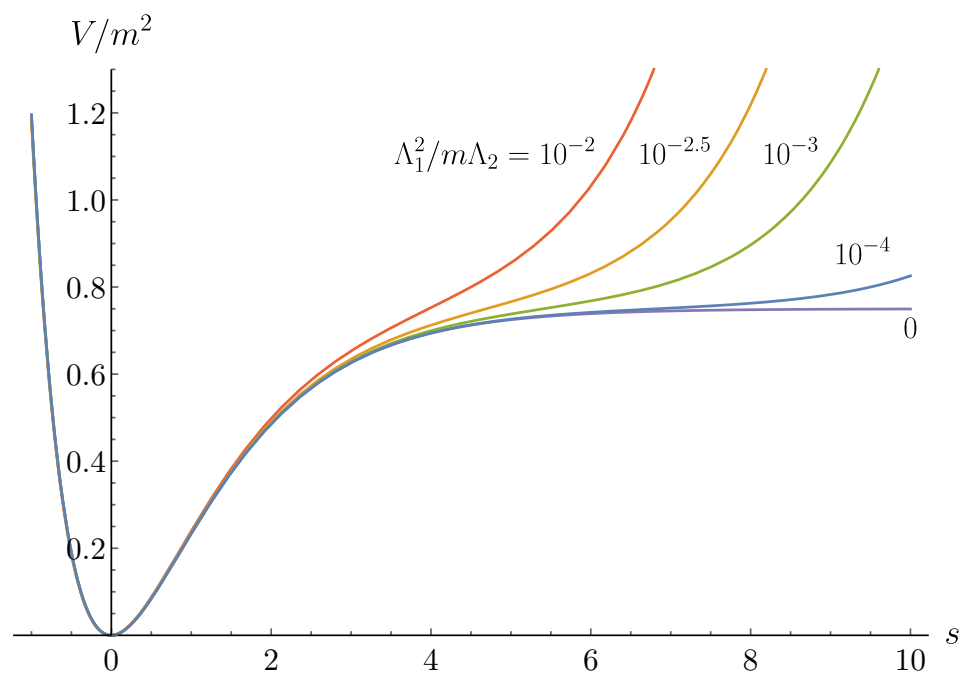

Figure 3: The effective inflationary potential (45) for different values of $\Lambda_{1}^{2} / m \Lambda_{2}$. The curve labeled $\Lambda_{1}^{2} / m \Lambda_{2}=0$ is the Starobinsky potential.

As was done in the previous Section, the system of equations (44) can be formally solved and substituted into (43) in order to obtain the $S$-dependent effective inflationary potential. However, once again the resulting expression is not particularly illustrative. Let us write schematically $\lambda_{8}^{00 i} S \sim \mu^{0 i} \sim \Lambda_{1}$ and $\lambda_{8}^{0 i j} S \sim \mu^{i j} \sim \Lambda_{2}$, so that $\left\langle\phi_{i}\right\rangle_{\text {inf }} \sim \Lambda_{1} / \Lambda_{2}$, and

$$
\Delta V_{\mathrm{inf}} \sim \frac{\sqrt{3} m \sinh (\sqrt{2 / 3} s)}{2(1+\tanh (s / \sqrt{6}))} \frac{\Lambda_{1}^{2}}{\Lambda_{2}} \sim m \frac{\sqrt{3} \Lambda_{1}^{2}}{8 \Lambda_{2}} e^{\sqrt{2 / 3} s} .
$$

Fig. 3 shows the form of the scalar potential (43) as a function of $\Lambda_{1}^{2} / m \Lambda_{2}$, demonstrating that the mixing parameters $\mu^{0 i}, \lambda_{8}^{00 i}$ need only be small compared to $\left(m \Lambda_{2}\right)^{1 / 2}$ in order to allow for inflation. Fig. 4 displays the corresponding CMB parameters $n_{s}$ and $r$, and compares them with the $68 \%$ and $95 \%$ CL limits from Planck and other data [21]. As in the previous cases, we see that $r$ lies within a factor $\sim 2$ of the Starobinsky prediction, far below the current upper limit, whereas $n_{s}$ lies beyond the Planck 95\% CL range for $\Lambda_{1}^{2} / m \Lambda_{2}<\left\{10^{-3.3}, 10^{-3.4}, 10^{-3.5}\right\}$ for $N_{*}=\left\{50, N_{*}^{\max }, 60\right\}$.

The curves shown in Fig. 3 must be taken with a pinch of salt, as the approximation (45) is only an order-of-magnitude estimate for the shape of the inflationary potential. In general, the couplings $\mu^{a b}$ and $\lambda_{8}^{a b c}$ will be unrelated, and the corresponding effective potential can acquire a more complicated structure. The potential can for example, begin to rise exponentially, or develop a secondary minimum thus preventing the successful realization of Starobinsky inflation. In Fig. 15, we show the form of the effective potential for an acceptable set of parameters where we have taken $\mu^{0 i}=\lambda_{8}^{00 i}=10^{-6}$ and a representative set of parameters in the range $\mu^{i j} \sim(0.1-0.8) M_{\mathrm{GUT}}, \lambda_{8}^{0 i j}$ and $\lambda_{8}^{i j k} \sim \pm(0.1-1)$. We see a simple valley structure in which the evolution of $s$ will lead to a standard Starobinsky-like 


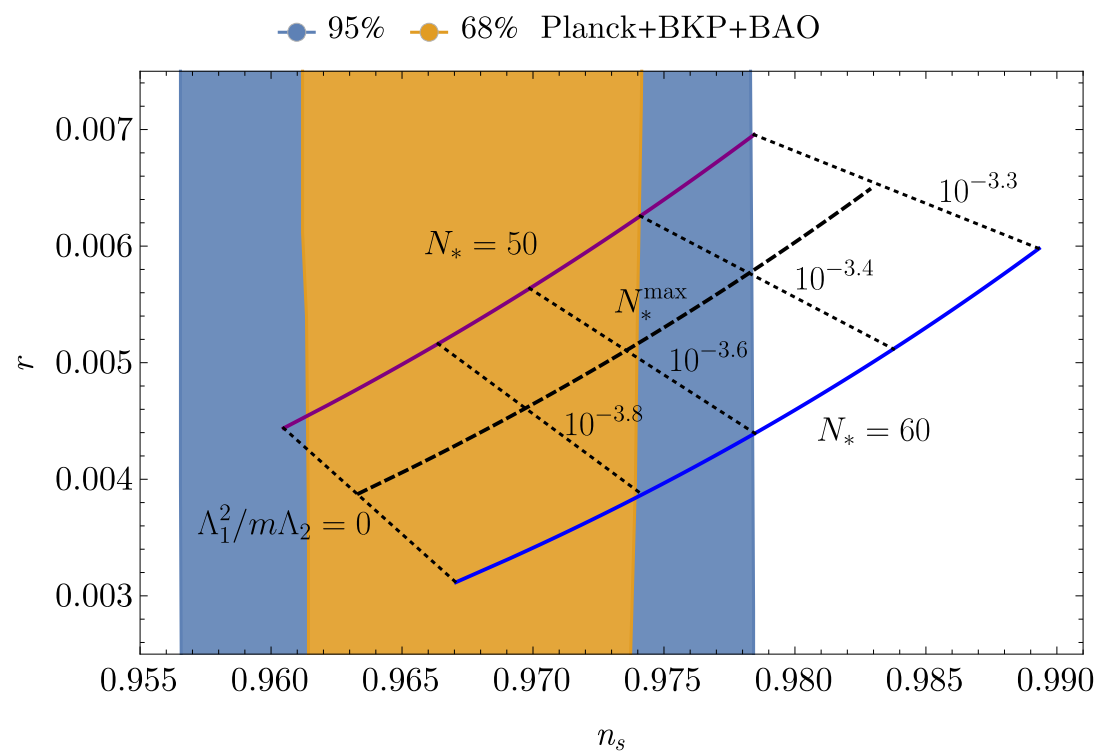

Figure 4: Parametric $\left(n_{s}, r\right)$ curves as functions of $\Lambda_{1}^{2} / m \Lambda_{2}$ for $N_{*}=50,60$ and $N_{*}^{\max } \simeq$ 53.3, with the 68\% and 95\% CL constraints from Planck and other data [21] shown in the background. The solid curves illustrate the parametric dependence using the analytical approximation (45). The dashed curve uses the analytical approximation (45) together with (108) to determine the dependence at $N_{*}^{\max }$. The dotted curves are for illustrative values of $\Lambda_{1}^{2} / m \Lambda_{2}$.

inflationary behavior, as discussed in more detail in the next subsection. Note that the evolution in Fig. 5 would appear to involve a change in direction of the fields in field space. In principle, this could have a significant effect on the final values of the anisotropy parameters $n_{s}$ and $r$ [50]. However, the field remains closely aligned with the instantaneous minimum and inflation proceeds as in the single field case, although this is not apparent in the figure, because of the range of scales plotted. There is no significant production of isocurvature perturbations, even for singlets $\phi_{i}$ lighter than the inflaton, as the no-scale structure naturally constrains the width of the inflationary valley so that during inflation, their masses are much larger than the Hubble scale as we already saw in Eq. (19) and as illustrated in Fig. 5 ,

After inflation ends, $S$ and the other singlets $\phi_{i}$ undergo damped oscillations. Assuming that during these oscillations the $\phi_{i}$ remain small, the evolution will track the 


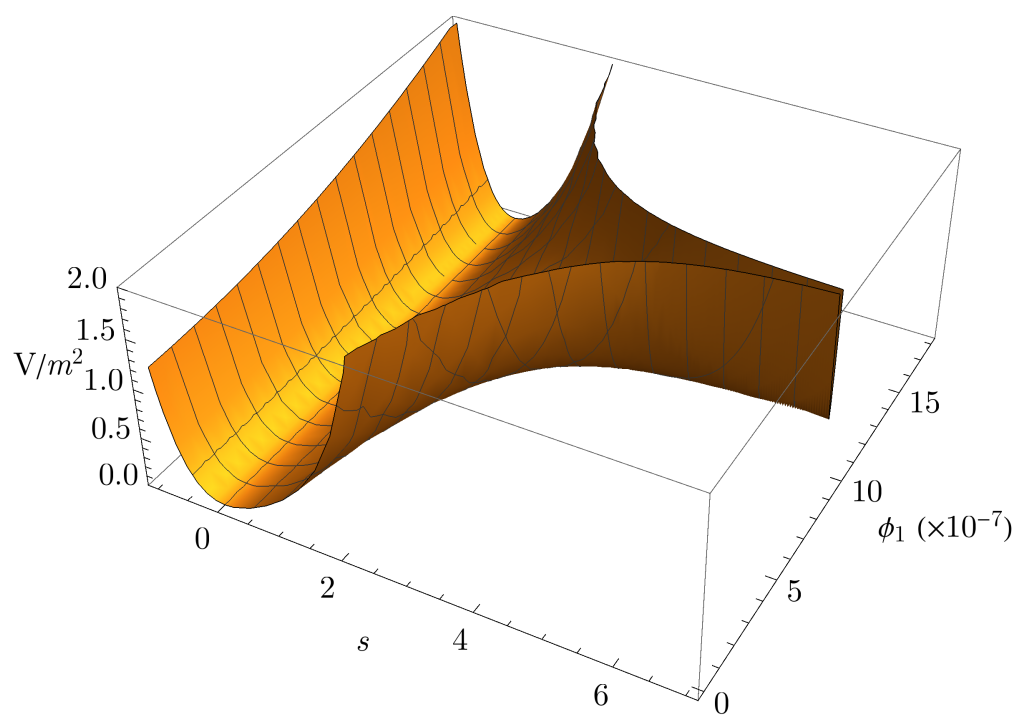

Figure 5: The effective inflationary potential for a representative set of parameters with $\mu^{i j} \sim(0.1-0.8) M_{\mathrm{GUT}}, \lambda_{8}^{0 i j}$ and $\lambda_{8}^{i j k} \sim \pm(0.1-1)$ and $\mu^{0 i}=\lambda_{8}^{00 i}=10^{-6}$. The singlets $\phi_{2}$ and $\phi_{3}$ are 'integrated out' numerically for every value of $s$. The evolution is seen to proceed to $s=0$, yielding Starobinsky-like inflation.

instantaneous solution to the system of equations

$$
\begin{aligned}
\frac{\partial V}{\partial \bar{\phi}^{i}} & \simeq 4 \sum_{j, k}\left(\bar{\mu}_{i k} \mu^{k j}+3 \bar{\mu}_{i k} \lambda_{8}^{k j 0} S+3 \bar{\lambda}_{8 i k 0} \mu^{j k} \bar{S}+9|S|^{2} \lambda_{8}^{0 j k} \bar{\lambda}_{80 i k}\right) \phi_{j} \\
& +2 S \sum_{j}\left(2 \bar{\mu}_{i j} \mu^{j 0}+3 \bar{\mu}_{i j} \lambda_{8}^{00 j} S+6 \mu^{0 j} \bar{\lambda}_{80 i j} \bar{S}+9 \bar{\lambda}_{80 i j} \lambda_{8}^{00 j}|S|^{2}\right)+\cdots
\end{aligned}
$$

Similarly to the case studied in the previous Section, this approximation may break down for $\lambda_{8}^{0 i j} \gtrsim \mu^{i j}$. When this is not the case, and if for simplicity we assume that $\mu^{0 i} \gtrsim \lambda_{8}^{00 i} 12$, the equations (46) can be solved trivially, resulting in the ratio

$$
\frac{\rho_{\phi_{i}}}{\rho_{s}} \sim m^{-2} \sum_{j} \bar{\mu}_{0 j} \mu^{0 j} .
$$

Therefore, reheating through inflaton decay requires $\mu^{0 i}<10^{-5}$.

\subsection{Numerical results}

In subsections 3.1 and 3.2 we have discussed the analytical constraints on the superpotential parameters $\mu^{0 i}$ and $\lambda_{8}^{00 i}$ that are imposed by the requirement of successful Starobinsky-like inflation. We now show numerical results that support these conclusions.

\footnotetext{
${ }^{12}$ Otherwise, if $\mu^{0 i} \lesssim \lambda_{8}^{00 i}$, the result reduces to $(35)$.
} 

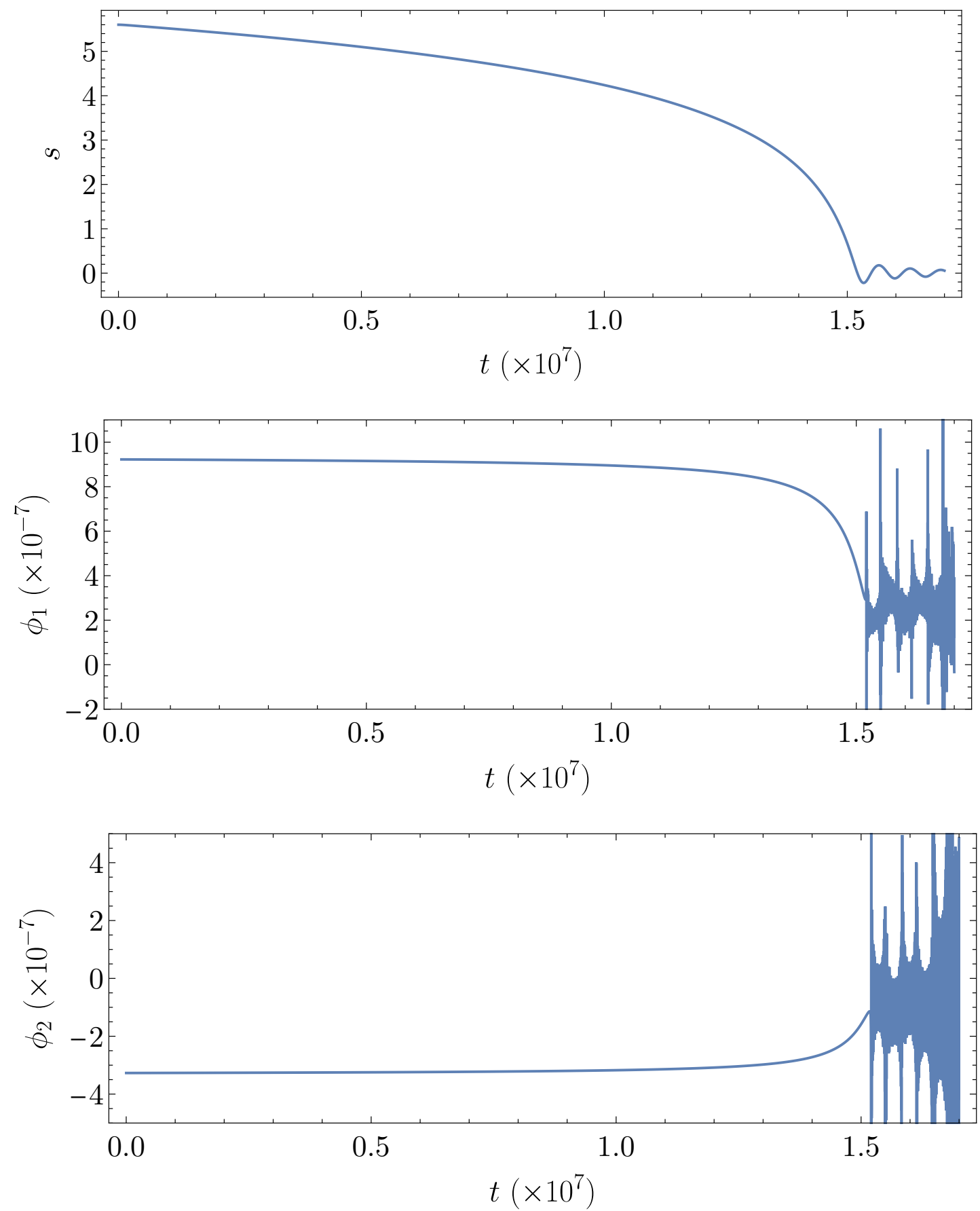

Figure 6: Evolution of the canonically-normalized inflaton $s$ and the SM singlet fields $\phi_{1}$ and $\phi_{2}$ during inflation, for $\mu^{0 i}=\lambda_{8}^{00 i}=10^{-6}$ and the same representative set of parameters as used in Fig. 5. The fields are assumed to start along the inflationary trajectory. 

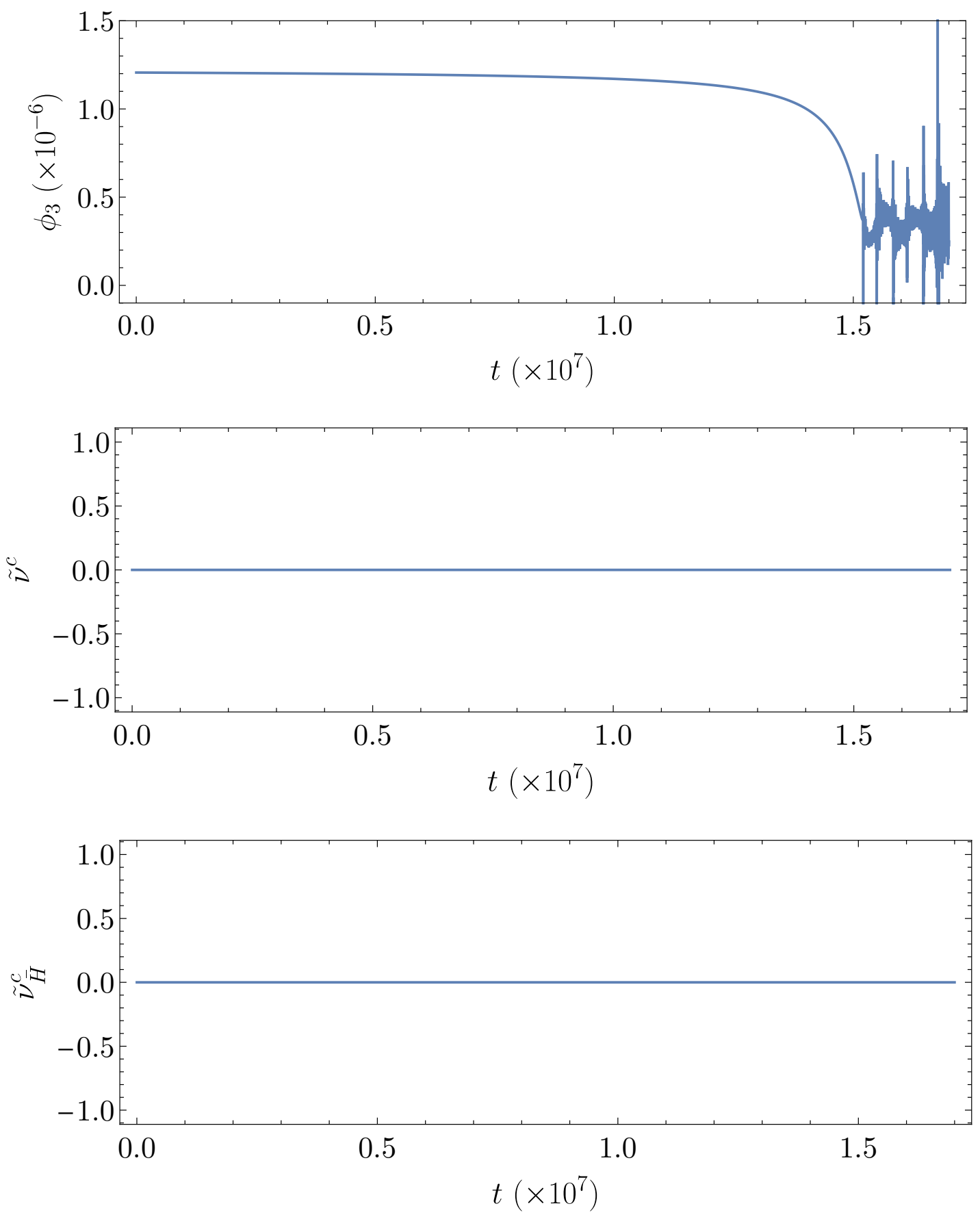

Figure 7: Evolution of the fields $\phi_{3}, \tilde{\nu}^{c}$ and $\tilde{\nu}_{\bar{H}}^{c}$ during inflation, also for $\mu^{0 i}=\lambda_{8}^{00 i}=10^{-6}$ and the same representative set of parameters as used in Fig. 5. The fields are again assumed to start along the inflationary trajectory. 
Figures 6 and 7 show the time evolutions of all the scalar singlet fields and $\tilde{\nu}^{c}, \tilde{\nu}_{\bar{H}}^{c}$ for $\mu^{0 i}=\lambda_{8}^{00 i}=10^{-6}$ and the same representative set of parameters as used in Fig. 5 , assuming that the fields start along the bottom of the inflationary 'valley' in field space. These results were obtained by numerical integration of the supergravity equations of motion:

$$
\ddot{\Psi}^{\alpha}+3 H \dot{\Psi}^{\alpha}+\Gamma_{\beta \gamma}^{\alpha} \dot{\Psi}^{\beta} \dot{\Psi}^{\gamma}+K^{\alpha \bar{\beta}} \frac{\partial V}{\partial \bar{\Psi}^{\bar{\beta}}}=0
$$

Here the indices run over all field components, with $\Psi^{\alpha} \equiv\left\{T, \phi_{a}, \tilde{\nu}^{c}, \tilde{\nu}_{\bar{H}}^{c}, \cdots\right\}, K^{\alpha \bar{\beta}}$ denotes the inverse Kähler metric, and the connection coefficients are given by

$$
\Gamma_{\beta \gamma}^{\alpha}=K^{\alpha \bar{\delta}} \partial_{\beta} K_{\gamma \bar{\delta}}
$$

It is clear from the evolution of $s$ in Fig. 6 that Starobinsky-like inflation is realized. Figs. 6 and 7 show that after the end of inflation the singlets $\phi_{i}$ undergo forced oscillations, with amplitudes much larger than the expected values assuming an adiabatic tracking of the inflaton value. This is an illustration of the phenomenon discussed around (33), namely that adiabaticity is violated when relatively large values for the parameters $\lambda_{8}^{0 i j}$ are chosen. As Fig. 8 demonstrates, after a few oscillations a significant fraction of the energy stored in the inflaton oscillations is transferred to the singlets $\phi_{i}$. The lower panel of Fig. 8 exhibits resonant enhancement of the $\phi_{2}$ amplitude when the solution of $\partial_{\phi_{i}} V=0$ is divergent, i.e., around the points where (46) cannot be inverted. As expected, the enhancement occurs when $s \ll 1$. This result confirms that strong segregation is a sufficient condition for reheating to occur through the decay of $s$.

Figs. 9 and 10 show the corresponding numerical results for a solution with the same parameters, but with a perturbed initial condition $\widetilde{\nu}_{\bar{H}}^{c}=5 \times 10^{-3}$. This perturbation seeds the oscillations of the remaining SM singlets, and drives an uphill roll of the inflaton due to the connection-dependent terms in (48), namely:

$$
\Gamma_{\alpha \beta}^{S} \dot{\Psi}^{\alpha} \dot{\Psi}^{\beta} \simeq-\frac{1}{2 \sqrt{3}} \sinh (\sqrt{2 / 3} s)\left(\dot{\phi}_{1}^{2}+\dot{\phi}_{2}^{2}+\dot{\phi}_{3}^{2}+\left(\dot{\tilde{\nu}}^{c}\right)^{2}+\left(\dot{\tilde{\nu}}_{\dot{H}}^{c}\right)^{2}\right)+\cdots
$$

As the value of $s$ increases, the oscillations of the fields are rapidly damped, and the subsequent evolution resembles that shown in Fig. 6 and Fig. 7, though with an increased total number of $e$-folds, and Starobinsky-like values of $\left(n_{s}, r\right)$.

Fig. 11 shows the constraints from Planck and other data [21] on the model in the $\left(\mu^{0 i}, \lambda_{8}^{00 i}\right)$ plane, calculated in a fully numerical fashion. Due to the large number of independent parameters, we have made the simplifying assumption $\mu^{0 i}=\mu^{0 i^{\prime}}$ for any $i, i^{\prime}$ (and similarly $\lambda_{8}^{00 i}=\lambda_{8}^{00 i^{\prime}}$ ). In the left panel the constraints are calculated for the same representative set of parameters as used in Fig. 5. We note that, e.g., the upper limit for $\mu^{0 i}$ is reasonably close to the analytical approximation $\Lambda_{1} \sim\left(10^{-3.3} m \Lambda_{2}\right)^{1 / 2}$ obtained in 

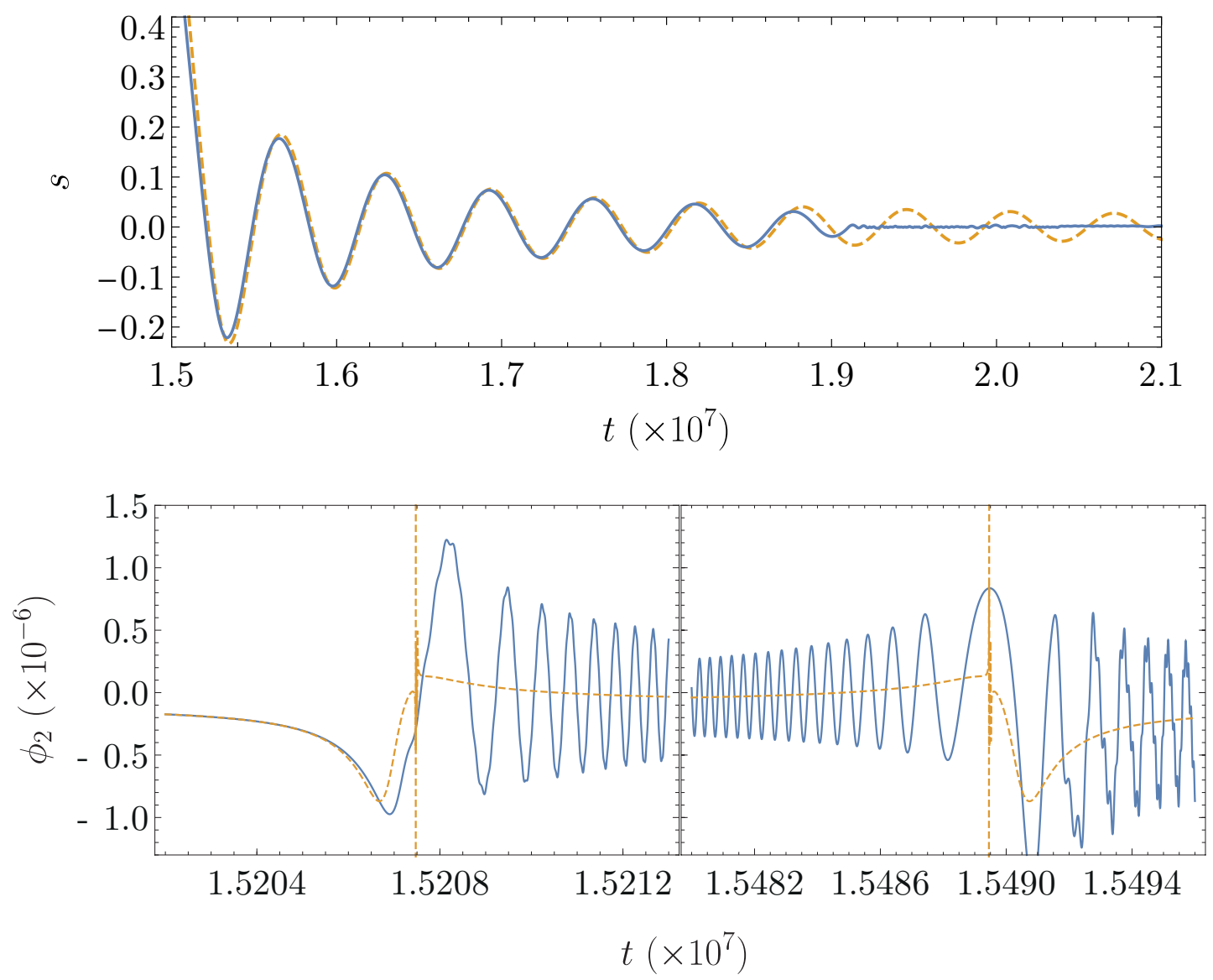

Figure 8: Evolution of the canonically-normalized inflaton s and the $S M$ singlet $\phi_{2}$ during reheating, for $\mu^{0 i}=\lambda_{8}^{00 i}=10^{-6}$ and the same representative set of parameters as used in Fig. 囵. Upper panel: evolution of the inflaton field s (blue, continuous), compared to the pure Starobinsky case (orange, dashed). Lower panel: evolution of the singlet field $\phi_{2}$ (blue, continuous), compared to the instantaneous solution of the equations $\partial_{\phi_{i}} V=0$ (orange, dashed). Notice the different horizontal scales in the two panels.

Fig. 4. In the right panel, the constraints correspond to the same representative set of parameters, but with the $\lambda_{8}^{i j k} \rightarrow 10^{-2} \lambda_{8}^{i j k}$. Here the solution is reasonably close to the analytical approximation (45). We have also verified that the $68 \%$ and $95 \%$ CL contours are unchanged under the assumption of multiple light states with $\mu^{i j} \lesssim 10^{-5}$.

\subsection{A symmetry argument for segregation}

As we have seen above, the parameters that cause mixing between the inflaton field $S$ and other singlet fields $\phi_{i}$, such as $\lambda_{8}^{00 i}$ and $\mu^{0 i}$, must be strongly suppressed in order to achieve successful inflation. In fact, such a suppression can naturally be obtained if one adopts an 

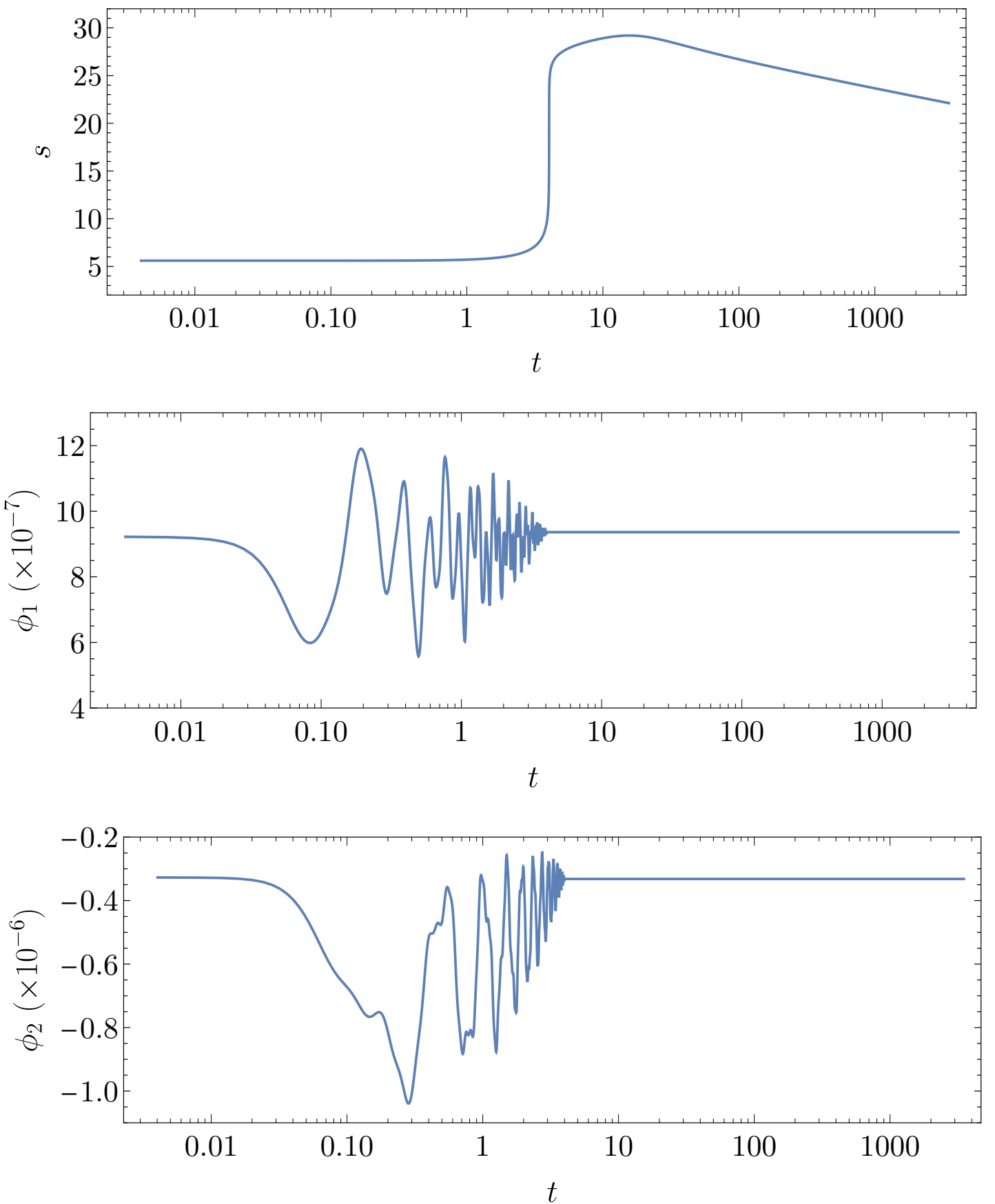

Figure 9: Evolution of the canonically-normalized inflaton $s$ and the SM singlets $\phi_{1}$ and $\phi_{2}$ during inflation, for $\mu^{0 i}=\lambda_{8}^{00 i}=10^{-6}$ and the same representative set of parameters as used in Fig. 5. The perturbed initial condition $\tilde{\nu}_{\bar{H}}^{c}=5 \times 10^{-3}$ is assumed. 

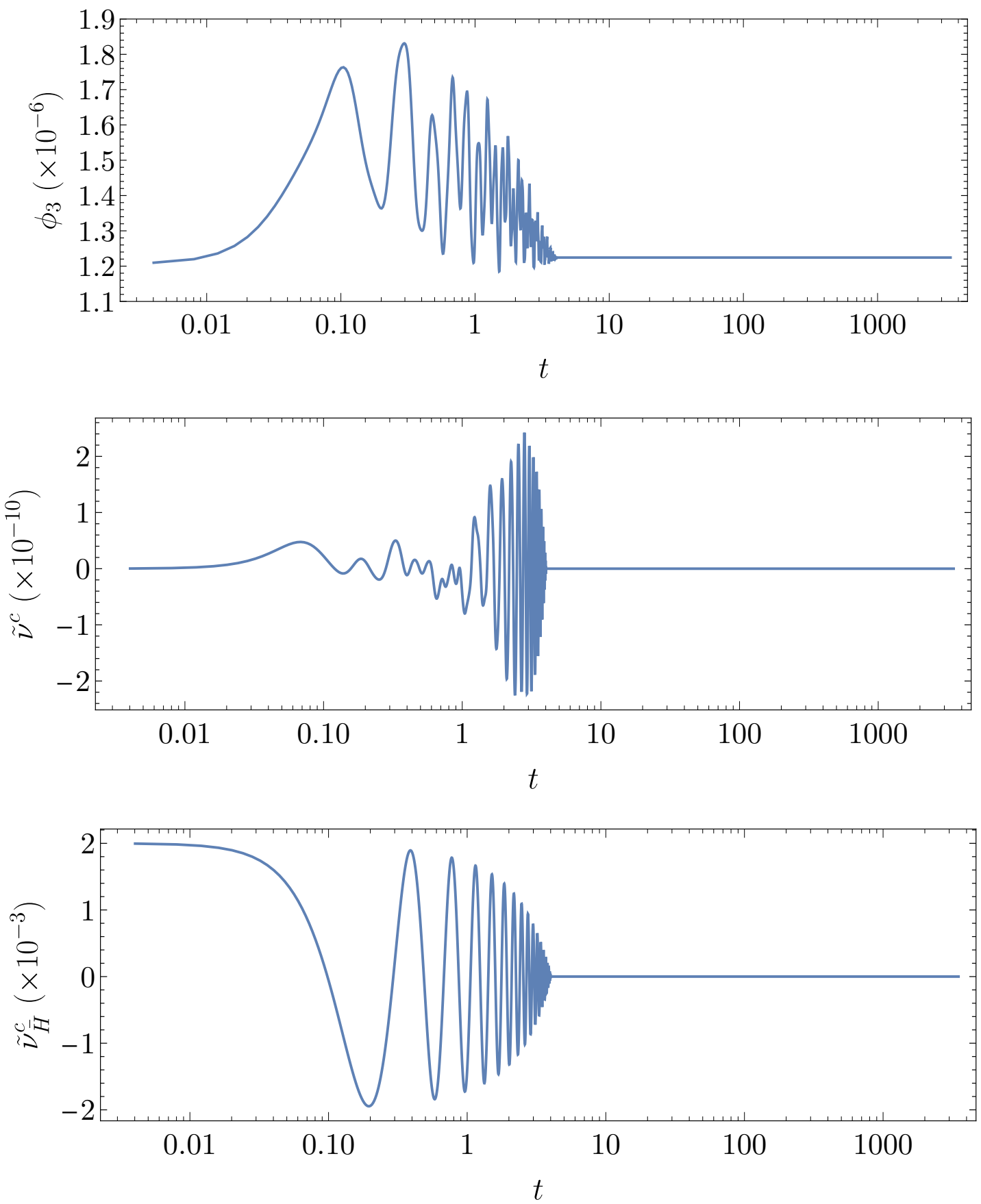

Figure 10: Evolution of the fields $\phi_{3}, \tilde{\nu}^{c}$ and $\tilde{\nu}_{\bar{H}}^{c}$ during inflation, also for $\mu^{0 i}=\lambda_{8}^{00 i}=10^{-6}$ and the same representative set of parameters as used in Fig. 5. The perturbed initial condition $\tilde{\nu}_{\bar{H}}^{c}=5 \times 10^{-3}$ is again assumed. 


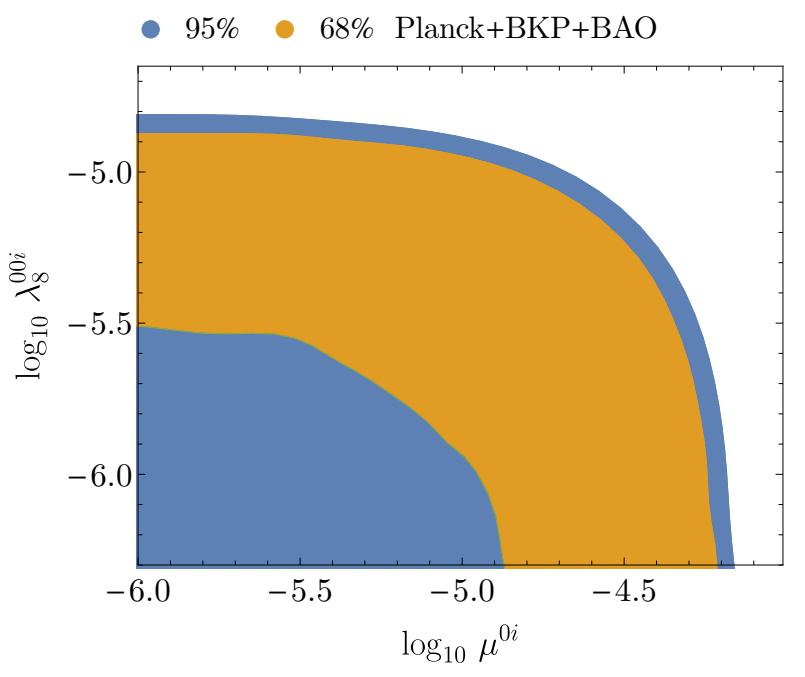

(a) $\mu^{i j} \lesssim 10^{-2}, \lambda_{8}^{i j k} \lesssim 1$

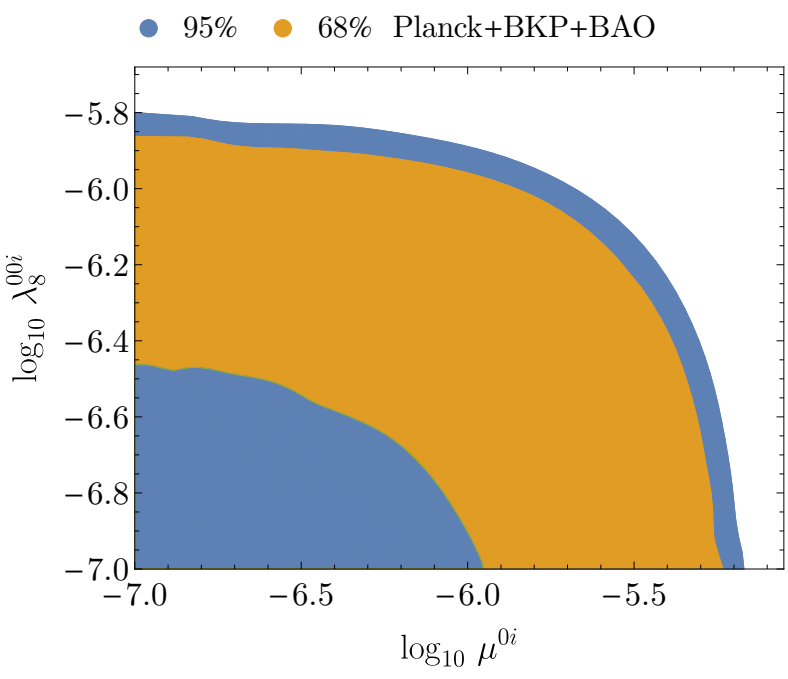

(b) $\mu^{i j}, \lambda_{8}^{i j k} \lesssim 10^{-2}$

Figure 11: The numerically-calculated $68 \%$ and $95 \%$ CL regions in the $\left(\mu^{0 i}, \lambda_{8}^{00 i}\right)$ plane at $N_{*}=50$ for the no-scale model with superpotential (5). Here for simplicity we have assumed that $\mu^{0 i}=\mu^{0 i^{\prime}}$ for any $i, i^{\prime}$, and similarly $\lambda_{8}^{00 i}=\lambda_{8}^{00 i^{\prime}}$.

appropriate definition of $R$-parity. Let us assign odd $R$-parity to the matter superfields $F$, $\bar{f}$, and $\ell^{c}$, and even $R$-parity to the Higgs multiplets $h$ and $\bar{h}$, as usual. We also assign $R$ parity even to $H, \bar{H}$, and $S$, while $\phi_{i}$ are assumed to be $R$-parity odd. With this assignment as well as the $\mathbb{Z}_{2}$ parity defined in (4), we can forbid the following couplings:

$$
\lambda_{6}^{i 0}=\lambda_{7}^{i}=\lambda_{8}^{00 i}=\lambda_{8}^{i j k}=\mu^{0 i}=0 .
$$

Note that with this $R$-parity assignment, the inflationary potential would be of the exact Starobinsky form given in (23). On the other hand, $\lambda_{8}^{0 i j}$ couplings are allowed by this $\mathbb{Z}_{2}$ symmetry, but as we have seen in our previous numerical results, taking these couplings of order unity does not adversely affect inflation. Thus, this $R$-parity assignment would be sufficient for obtaining the necessary strong segregation. We also note that this $R$-parity is not broken by the vevs of $H$ and $\bar{H}$ since they are $R$-parity even. Therefore, this $R$-parity is respected at low energies unless $\phi_{i}$ acquire vevs 13

\section{Reheating Constraints}

In this section, we study the neutrino mass structure in this model and discuss its connection to reheating after inflation. As we see below, neutrino mass terms are provided by Yukawa

\footnotetext{
${ }^{13}$ This $R$-parity assignment differs from that used in [31. With this assignment $R$-parity is exact unless some of the couplings in (51) are turned on.
} 
couplings in the superpotential. In GUTs, these Yukawa couplings may be related to other Yukawa couplings - thus, we first discuss Yukawa unification in our model in Section 4.1. We then study the neutrino mass structure in Section 4.2 and its connection to reheating dynamics in Section 4.3 .

\subsection{Yukawa unification}

The Yukawa coupling terms in the low-energy effective theory of this model are given by

$$
W_{\text {Yukawa }}=f_{u} h_{u} Q \bar{u}+f_{\nu} h_{u} L \nu_{R}^{c}-f_{d} h_{d} Q \bar{d}-f_{e} h_{d} L \bar{e},
$$

where we have suppressed generation indices for simplicity. In ordinary SU(5) GUTs, we expect

$$
f_{d}\left(M_{\mathrm{GUT}}\right)=f_{e}\left(M_{\mathrm{GUT}}\right)
$$

at the GUT scale. For the third generation (bottom and tau) Yukawa couplings, this relation is satisfied at the $\mathcal{O}(10) \%$ level. For the first two generations, however, there are $\mathcal{O}(1)$ differences. Such deviations may be explained by means of higher-dimensional operators suppressed by the Planck-scale [51] or higher-dimensional Higgs representations [52] within the framework of SU(5) GUTs.

In the case of flipped $\mathrm{SU}(5) \times \mathrm{U}(1)$, on the other hand, we have

$$
f_{u}\left(M_{\mathrm{GUT}}\right)=f_{\nu}\left(M_{\mathrm{GUT}}\right)
$$

as these two Yukawa couplings come from the same $\lambda_{2}$ term in (3). In this case, the downtype Yukawa coupling $f_{d}$, which is matched onto $\lambda_{1}$, is unrelated to the charged lepton Yukawa coupling $f_{e}$, which originates from $\lambda_{3}$. Therefore, the less successful prediction (53) for the first two generations in ordinary SU(5) GUTs is not problematic in flipped $\mathrm{SU}(5) \times \mathrm{U}(1)$ models. As we see below, even though we have the unification condition (54) for $f_{\nu}$, we can explain the observed pattern of neutrino mass differences and mixing angles by choosing $\lambda_{6}^{i a}$ and $\mu^{a b}$ appropriately.

\subsection{Neutrino masses}

Next, let us investigate the neutrino mass matrix in flipped $\mathrm{SU}(5) \times \mathrm{U}(1)$. After $h, \bar{h}$, $H$, and $\bar{H}$ develop vevs, the Yukawa terms $\lambda_{2}$ and $\lambda_{6}$ lead to Dirac mass terms for $\nu, \nu^{c}$, and $\tilde{\phi}_{a}$, where $\tilde{\phi}_{a}$ denotes the fermionic component of $\phi_{a}$. If the singlet fields $\phi_{a}$ acquire vevs, the Higgsino may also mix with right-handed neutrinos via the $\lambda_{6}^{i a}$ couplings, which results in the $R$-parity violation. The $R$-parity violating effects may also be induced by Higgsino-singlet mixing via the $\lambda_{7}^{a}$ couplings. Here, we focus on the following two cases 
where there is no (strong) $R$-parity violation: (A) No singlet field develops a vev, $\left\langle\phi_{a}\right\rangle=0$, and the inflaton $S=\phi_{0}$ does not participate in the neutrino mass generation. This setup is realized when $\lambda_{6}^{i 0}=\lambda_{7}^{i}=0$ (though $\lambda_{7}^{0}$ is allowed). Note that this scenario requires at least four singlets, our default assumption. (B) One of the $\phi_{i}$ fields (denoted by $\phi_{i^{\prime}}$ ) acquires a non-zero vev. If this field is responsible for the $\mu$ term, its $R$-parity must be positive, and thus does not couple to the neutrino sector. Instead of this $\phi_{i^{\prime}}$, the inflaton $S$ plays a role in neutrino mass generation. Thus only $\lambda_{7}^{i^{\prime}}$ is non-zero and in particular, $\lambda_{7}^{0}=0$. In the case of three singlets, since the $R$-parity of all three singlets must be negative, $\lambda_{7}=0$ for all three, and a GM term is necessary to produce a $\mu$ term. Note that in this case, there is some $R$-parity violation due to the presence of both quadratic and cubic superpotential terms, though the $R$-parity violation is weakly transmitted to the matter sector, and the lifetime of the lightest supersymmetric particle (LSP) remains sufficiently long [11]. In what follows, we study separately the neutrino mass structure and reheating dynamics for these two scenarios (A) and (B).

\subsubsection{Scenario (A): inflaton decouples from the neutrino sector}

Here we assume $\left\langle\phi_{a}\right\rangle=0$, which is achieved when all of the $\mu^{a b}$ are much larger than the supersymmetry-breaking scale. In this case, we need to introduce a Giudice-Masiero term to obtain the MSSM Higgs $\mu$-term. We further assume

$$
\lambda_{6}^{i 0}=\lambda_{7}^{i}=\mu^{0 i}=0
$$

which is assured by the $R$-parity discussed in Section 3.4. This prevents the fermionic component of $\phi_{0}$ from mixing with neutrinos. The mass matrix for $\nu_{i}, \nu_{i}^{c}$, and $\tilde{\phi}_{i}$ is then given by [5] 7 ]

$$
\mathcal{L}_{\text {mass }}^{(\nu)}=-\frac{1}{2}\left(\begin{array}{lll}
\nu_{i} & \nu_{i}^{c} & \tilde{\phi}_{i}
\end{array}\right)\left(\mathcal{M}_{\nu}\right)_{i j}\left(\begin{array}{c}
\nu_{j} \\
\nu_{j}^{c} \\
\tilde{\phi}_{j}
\end{array}\right)+\text { h.c. },
$$

with

$$
\left(\mathcal{M}_{\nu}\right)_{i j} \equiv\left(\begin{array}{ccc}
0 & \lambda_{2}^{i j}\left\langle\bar{h}_{0}\right\rangle & 0 \\
\lambda_{2}^{T i j}\left\langle\bar{h}_{0}\right\rangle & 0 & \lambda_{6}^{i j}\left\langle\tilde{\nu}_{\bar{H}}^{c}\right\rangle \\
0 & \lambda_{6}^{T i j}\left\langle\tilde{\nu}_{\bar{H}}^{c}\right\rangle & 2 \mu^{i j}
\end{array}\right),
$$

where we have used two-component notation. The mass matrix $\mathcal{M}_{\nu}$ is a complex symmetric matrix and thus can be diagonalized with a unitary matrix. By using $\left|\lambda_{2}^{i j}\left\langle\bar{h}_{0}\right\rangle\right| \ll$ $\left|\lambda_{6}^{i j}\left\langle\tilde{\nu}_{\bar{H}}^{c}\right\rangle\right|,\left|\mu^{i j}\right|$, we obtain the following mass matrix for the three light active neutrinos:

$$
\mathcal{M}_{L} \simeq \frac{2\left\langle\bar{h}_{0}\right\rangle^{2}}{\left\langle\tilde{\nu}_{\bar{H}}^{c}\right\rangle^{2}}\left[\lambda_{2}\left(\lambda_{6}^{T}\right)^{-1} \mu \lambda_{6}^{-1} \lambda_{2}^{T}\right]
$$


Thus, light neutrino masses can naturally be explained by the (double) seesaw mechanism [53, 54]. Even though the structure of the matrix $\lambda_{2}$ is related to the up-quark Yukawa matrix through the unification relation (54), we still have a sufficient number of degrees of freedom in the matrices $\lambda_{6}$ and $\mu$, and thus can easily find a form of $\mathcal{M}_{L}$ that fits the neutrino oscillation data. The couplings $\lambda_{6}^{i j}$ and $\mu^{i j}$ in general contain extra CP phases, and non-zero $\mu^{i j}$ cause lepton-number violation. As a consequence, this model may explain baryon asymmetry of the Universe via thermal leptogenesis [55, 56] 14

Finally, the mass matrices of heavier states are given by

$$
\mathcal{M}_{H}=\left(\begin{array}{cc}
0 & \lambda_{6}^{i j}\left\langle\tilde{\nu}_{\bar{H}}^{c}\right\rangle \\
\lambda_{6}^{T i j}\left\langle\tilde{\nu}_{\bar{H}}^{c}\right\rangle & 2 \mu^{i j}
\end{array}\right) .
$$

If furthermore, $\lambda_{6}^{i j}\left\langle\tilde{\nu}_{\bar{H}}^{c}\right\rangle \ll \mu^{i j}$, then the corresponding heavy mass eigenvalues are of order $\left(\lambda_{6}^{i j}\left\langle\tilde{\nu}_{\bar{H}}^{c}\right\rangle\right)^{2} / \mu^{i j}$ and $\mu^{i j}$.

\subsubsection{Scenario (B): The inflaton couples to the neutrino sector}

Next, we discuss the case where a combination of the singlet fields $\phi_{i}$, called $\phi_{i^{\prime}}$, acquires a vev: $\left\langle\phi_{i^{\prime}}\right\rangle \neq 0$. We assume that this singlet field does not have a coupling to $F_{i}$, in order to suppress $R$-parity violation: $\lambda_{6}^{i i^{\prime}}=0$. In this case, the $\lambda_{7}^{i^{\prime}}$ term leads to the MSSM $\mu$-term, $\mu=\lambda_{7}^{i^{\prime}}\left\langle\phi_{i^{\prime}}\right\rangle$. To obtain three massive active neutrinos, we instead couple the inflaton field to $F_{i}: \lambda_{6}^{i 0} \neq 0$. We then suppress the $\lambda_{7}^{0}$ coupling to avoid $R$-parity violation.

For $i, j \neq i^{\prime}$, the neutrino mass matrix has the same structure as $\mathcal{M}_{\nu}$ in (57), and thus light neutrino masses for these generations are again given by (158) 15. For $i=j=i^{\prime}$, on the other hand, the mass matrix is given by

$$
\mathcal{L}_{\text {mass }}^{\left(i^{\prime}\right)}=-\frac{1}{2}\left(\begin{array}{lll}
\nu_{i^{\prime}} & \nu_{i^{\prime}}^{c} & \tilde{S}
\end{array}\right)\left(\begin{array}{ccc}
0 & \lambda_{2}^{i^{\prime} i^{\prime}}\left\langle\bar{h}_{0}\right\rangle & 0 \\
\lambda_{2}^{i^{\prime} i^{\prime}}\left\langle\bar{h}_{0}\right\rangle & 0 & \lambda_{6}^{i^{\prime} 0}\left\langle\tilde{\nu}_{\bar{H}}^{c}\right\rangle \\
0 & \lambda_{6}^{i^{\prime} 0}\left\langle\tilde{\nu}_{\bar{H}}^{c}\right\rangle & m
\end{array}\right)\left(\begin{array}{c}
\nu_{i^{\prime}} \\
\nu_{i^{\prime}}^{c} \\
\tilde{S}
\end{array}\right)+\text { h.c. },
$$

where $\tilde{S}$ denotes the fermionic partner of the inflaton $S$. The light mass eigenvalue for this mass matrix is then given by

$$
m_{\nu_{i^{\prime}}} \simeq \frac{m\left(\lambda_{2}^{i^{\prime} i^{\prime}}\left\langle\bar{h}_{0}\right\rangle\right)^{2}}{\left(\lambda_{6}^{i^{\prime} 0}\left\langle\tilde{\nu}_{\bar{H}}^{c}\right\rangle\right)^{2}},
$$

\footnotetext{
${ }^{14}$ As we discuss in Section 4.3.1, in this scenario the inflaton does not decay directly into heavy neutrinos, leaving thermal leptogenesis as a possibility. For this to occur, we need a high reheating temperature, and thus the strong reheating case discussed in Section 5.3 .2 is favored in this scenario.

${ }^{15}$ Here, we neglect the effects of $\lambda_{6}^{i 0}$ and $\mu^{i 0}$ for simplicity. The generalization to non-zero $\lambda_{6}^{i 0}$ and $\mu^{i 0}$ is straightforward. We also neglect mixing among generations, which is expected to be sizable according to neutrino oscillation data, to simplify the expressions, but the generalization is again straightforward. For more concrete expressions, see Ref. [7].
} 
while the heavier eigenvalues have masses

$$
m_{N_{i^{\prime} 1,2}}=\frac{1}{2}\left[m \mp \sqrt{\left(2 \lambda_{6}^{i^{\prime} 0}\left\langle\tilde{\nu}_{\bar{H}}^{c}\right\rangle\right)^{2}+m^{2}}\right],
$$

where $N_{i^{\prime} 1}$ and $N_{i^{\prime} 2}$ are $\nu_{i^{\prime}}^{c}$ and $\tilde{S}$-like states, respectively. For $m \ll \lambda_{6}\left\langle\tilde{\nu}_{\bar{H}}^{c}\right\rangle$, these two states form a pseudo-Dirac state with mass $\lambda_{6}\left\langle\tilde{\nu}_{\bar{H}}^{c}\right\rangle$ with splitting of order $m$. It is interesting to note the role played by the inflaton mass, $m$ for neutrino masses 16 . The light (mostly left-handed) neutrino masses are proportional to the inflaton mass, whilst the heavy state masses are split by the inflaton mass.

The part of the superpotential relevant for the $\nu_{i^{\prime}}^{c}$ and $S$ couplings can be written as

$$
W=\lambda_{2}^{i^{\prime} j} \nu_{i^{\prime}}^{c} L_{j} h_{u}+\lambda_{6}^{i / 0} \nu_{i^{\prime}}^{c} \nu_{\bar{H}}^{c} S+\frac{m}{2} S^{2} .
$$

Rotating the $\nu_{i^{\prime}}^{c}$ and $S$ fields into the mass eigenstates:

$$
\left(\begin{array}{l}
N_{i^{\prime} 1} \\
N_{i^{\prime} 2}
\end{array}\right)=\left(\begin{array}{cc}
\cos \theta & \sin \theta \\
-\sin \theta & \cos \theta
\end{array}\right)\left(\begin{array}{c}
\tilde{\nu}_{i^{\prime}}^{c} \\
S
\end{array}\right),
$$

with

$$
\tan 2 \theta=-\frac{2 \lambda_{6}^{i^{\prime} 0}\left\langle\tilde{\nu}_{\bar{H}}^{c}\right\rangle}{m}
$$

the superpotential (63) can then be expressed as

$$
W=\lambda_{2}^{i^{\prime} j}\left(\cos \theta N_{i^{\prime} 1}-\sin \theta N_{i^{\prime} 2}\right) L_{j} h_{u}+\frac{1}{2} m_{N_{i^{\prime} 1}} N_{i^{\prime} 1}^{2}+\frac{1}{2} m_{N_{i^{\prime} 2}} N_{i^{\prime} 2}^{2},
$$

where the masses $m_{N_{i^{\prime} 1,2}}$ are given in (62).

As we see below, the neutrino mass structure in this scenario is restricted by the constraint on the reheating temperature. We will discuss the compatibility of this constraint with the observed neutrino oscillation data in Section 4.3.2.

\subsection{Singlet decays}

Now we consider inflaton decay in the two scenarios discussed in the previous subsection. In Scenario (A), the inflaton does not couple to the neutrino sector, so at the tree level it can decay only into Higgs bosons and Higgsinos. In Scenario (B), on the other hand, the inflaton $S$ does couple to right-handed neutrinos but its coupling to the MSSM Higgs fields is suppressed in order to evade $R$-parity violation. We will find that there is a tight connection between neutrino masses and reheating dynamics in this case.

\footnotetext{
${ }^{16}$ The parameter $m$ is inflaton mass during inflation and reheating when the GUT symmetry remains exact. After GUT symmetry breaking the scalars associated with the inflaton multiplet receive GUT scale masses proportional to $\lambda_{6}\left\langle\tilde{\nu}_{\bar{H}}^{c}\right\rangle$.
} 


\subsubsection{Scenario (A): inflaton decay into Higgs/Higgsino}

Assuming (55), the superpotential couplings relevant to the inflaton decay are given by

$$
W_{S \text { decay }}=\lambda_{1}^{i j} F_{i} F_{j} h+\lambda_{2}^{i j} F_{i} \bar{f}_{j} \bar{h}+\lambda_{3}^{i j} \bar{f}_{i} \ell_{j}^{c} h+\lambda_{7}^{0} S h \bar{h}+3 \lambda_{8}^{0 i j} S \phi_{i} \phi_{j}+\frac{m}{2} S^{2} .
$$

If the mass eigenvalues of the mass matrix (59) are larger than the inflaton mass $m$, then the inflaton decay via the couplings $\lambda_{8}^{0 i j}$ is suppressed by a small light-heavy neutrino mixing angle of $\mathcal{O}\left(\lambda_{2}\left\langle\bar{h}_{0}\right\rangle /\left(\mu, \lambda_{6}\left\langle\tilde{\nu}_{\bar{H}}^{c}\right\rangle\right)\right)$, and thus is negligible. The $\lambda_{7}^{0}$ coupling gives rise to the inflaton-Higgs/Higgsino interactions:

$$
\mathcal{L}_{\text {int }}=-\frac{\lambda_{7}^{0}}{\sqrt{2}} s \tilde{h}_{u} \tilde{h}_{d}-\frac{m^{*} \lambda_{7}^{0}}{\sqrt{2}} s h_{u} h_{d}+\text { h.c. }
$$

which yields the following singlet decay rate:

$$
\Gamma\left(s \rightarrow \tilde{h}_{u} \tilde{h}_{d}\right)=\Gamma\left(s \rightarrow h_{u} h_{d}\right) \simeq \frac{\left|\lambda_{7}^{0}\right|^{2}}{8 \pi}|m| .
$$

The cross terms between the $\lambda_{1,2,3}$ and $\lambda_{7}$ terms in (67) also induce singlet-sfermion couplings. These couplings give rise to either three-body decay or two-body decay suppressed by the Higgs vev $\left\langle h^{0}\right\rangle$. Hence, these sfermion decay channels are sub-dominant.

As we discuss in Section 5.1, an upper limit on the inflaton decay rate is given by the over-production of gravitinos, which restricts the coupling $\lambda_{7}^{0}$ as 17

$$
\left|\lambda_{7}^{0}\right| \lesssim 10^{-5} \Delta
$$

though it could be substantially smaller. Since the inflaton plays no role in the neutrino mass generation, this limit has no implication for the neutrino mass structure, contrary to Scenario (B) discussed below.

\subsubsection{Scenario (B): inflaton decay into neutrinos}

In this case, the $\lambda_{7}^{0}$ coupling is set to be zero to avoid $R$-parity violation. Thus, the Higgs/Higgsino decay modes of the inflaton are suppressed. Instead, the inflaton couples to the neutrino sector through the $\lambda_{6}^{i^{\prime} 0}$ coupling, and thus can decay into a lepton and a Higgsino, or a slepton and a Higgs boson. Other decay channels are three-body decay processes or those dependent on a small vev, $\left\langle\phi_{i^{\prime}}\right\rangle$ or $\left\langle h^{0}\right\rangle$, and are thus subdominant.

\footnotetext{
${ }^{17}$ The constant $\Delta \geq 1$ parametrizes any dilution of the gravitino relic density posterior to reheating, due to the entropy increase produced by the decay of a long-lived particle (see Section 5.3.3).
} 
The relevant interactions are readily obtained from the superpotential (66), from which we evaluate the decay rates of the $s$-like state $\operatorname{Re}\left(\sqrt{2} N_{i^{\prime} 2}\right)$ :

$$
\Gamma\left(s \rightarrow L_{j} \tilde{h}_{u}\right)=\Gamma\left(s \rightarrow \tilde{L}_{j} h_{u}\right) \simeq \frac{\left|\lambda_{2}^{i^{\prime} j} \sin \theta\right|^{2}}{8 \pi} m_{N_{i^{\prime} 2}} .
$$

We then obtain a constraint on $\sin \theta$ as in (70):

$$
\left|\lambda_{2}^{i^{\prime} j} \sin \theta\right| \lesssim 10^{-5} \Delta
$$

We now discuss the implication of this constraint for light neutrino masses. We work on the basis where $\lambda_{2}$ is diagonalized. We first consider the case $i^{\prime}=3$, where $\lambda_{2}^{33} \simeq m_{t} /\left\langle\bar{h}^{0}\right\rangle \simeq 1$. In this case, the constraint (르) leads to

$$
10^{-5} \Delta \gtrsim|\sin \theta| \simeq\left|\frac{\lambda_{6}^{30}\left\langle\tilde{\nu}_{\bar{H}}^{c}\right\rangle}{m}\right|
$$

With (61), this bound gives

$$
m_{\nu_{3}} \gtrsim 10^{10} \cdot \frac{m_{t}^{2}}{m} \Delta^{-2} \sim 10 \mathrm{GeV} \Delta^{-2},
$$

which, in the absence of significant entropy production, is much larger than the current experimental limit from the Lyman $\alpha$ forest power spectrum obtained by BOSS in combination with the Planck $2015 \mathrm{CMB}$ data [57]: $\sum_{\nu} m_{\nu}<0.12 \mathrm{eV}$.

In the $i^{\prime}=2$ case, although the bound is relaxed by a factor of $10^{-4}$, the resultant neutrino mass value is still above this limit. In the $i^{\prime}=1$ case, however, $\lambda_{2}^{11} \simeq m_{u} /\left\langle\bar{h}^{0}\right\rangle \simeq$ $10^{-5}$, and thus the constraint (72) gives no limit on the mixing angle $\theta$. In this case, the neutrino mass is given by

$$
m_{\nu_{1}} \simeq 10^{-9} \times\left(\frac{m}{3 \times 10^{13} \mathrm{GeV}}\right)\left(\frac{\left|\lambda_{6}^{10}\right|}{10^{-3}}\right)^{-2}\left(\frac{\left|\left\langle\tilde{\nu}_{\bar{H}}^{c}\right\rangle\right|}{10^{16} \mathrm{GeV}}\right)^{-2} \mathrm{eV},
$$

which evades the experimental limit.

Recent global fits to neutrino oscillation data give [58]

$$
\begin{aligned}
\left|\delta m^{2}\right| & \equiv\left|m_{\nu_{2}}^{2}-m_{\nu_{1}}^{2}\right| \simeq 7.4 \times \mid 10^{-5} \mathrm{eV}^{2} \\
\left|\Delta m^{2}\right| & \equiv\left|m_{\nu_{3}}^{2}-\left(m_{\nu_{2}}^{2}+m_{\nu_{1}}^{2}\right) / 2\right| \simeq 2.5 \times 10^{-3} \mathrm{eV}^{2} .
\end{aligned}
$$

These values as well as the result in (75) indicate that, unless $\left|\lambda_{6}^{10}\left\langle\tilde{\nu}_{\bar{H}}^{c}\right\rangle\right|$ is extremely small, a Normal Hierarchy (NH) mass spectrum, i.e., $m_{\nu_{1}} \ll m_{\nu_{2}}<m_{\nu_{3}}$, is favored in this model. The other light neutrino masses in this case are predicted to be

$$
\begin{aligned}
& m_{\nu_{2}} \simeq\left|\delta m^{2}\right|^{\frac{1}{2}} \simeq 9 \times 10^{-3} \mathrm{eV}, \\
& m_{\nu_{3}} \simeq\left|\Delta m^{2}\right|^{\frac{1}{2}} \simeq 5 \times 10^{-2} \mathrm{eV} .
\end{aligned}
$$

We can easily obtain these values by choosing appropriately $\mu$ and $\lambda_{6}$ in (58): reheating does not impose significant restrictions for these two generations. 


\section{Post-Inflation}

\section{$5.1 \quad$ Reheating}

The temperature of the Universe following inflation depends on the inflaton decay rate, which we parameterize as $\Gamma_{s}=|y|^{2} m / 8 \pi$. For case (A), $y=\sqrt{2} \lambda_{7}^{0}$, and for case (B), $y=\sqrt{2} \lambda_{2}^{i^{\prime} j} \sin \theta$. This decay rate is bounded by the upper limit on the density of gravitinos produced in the relativistic plasma arising from the inflaton decay products [15,59 77]. BigBang Nucleosynthesis (BBN) imposes tight constraints on the decay rate of the inflaton for small gravitino masses $[67,72,73,77,80]$. However, if one assumes that the gravitino is sufficiently heavy to decay before BBN [79, 80], the dominant bound on its abundance comes from its contribution to the cold dark matter relic density. Assuming a present dark matter density $\Omega_{\text {cold }} h^{2}=0.12$ [21] and a standard thermal history with no post-reheating entropy production, we find the following constraint on the inflaton decay coupling $y$ [15]:

$$
|y|<2.7 \times 10^{-5}\left(1+0.56 \frac{m_{1 / 2}^{2}}{m_{3 / 2}^{2}}\right)^{-1}\left(\frac{100 \mathrm{GeV}}{m_{\mathrm{LSP}}}\right),
$$

implying that $\Gamma_{s} \lesssim 900 \mathrm{GeV}$ for $m=3 \times 10^{13} \mathrm{GeV}$. When the limit on $y$ is saturated, the relic density of the LSP is obtained by the non-thermal decay of the gravitino. For smaller $y$, the LSP abundance from decay is reduced and other mechanisms (such as freeze-out or coannihilations) must be operating so as to give the correct cold dark matter density.

Equivalently, in terms of the reheating temperature,

$$
T_{\text {reh }} \equiv\left(\frac{40}{g_{\text {reh }} \pi^{2}}\right)^{1 / 4}\left(\Gamma_{s} M_{P}\right)^{1 / 2} \lesssim\left(\frac{915 / 4}{g_{\text {reh }}}\right)^{1 / 4}\left(1.7 \times 10^{10} \mathrm{GeV}\right),
$$

where $g_{\text {reh }}$ is the number of relativistic degrees of freedom at $T_{\text {reh }}$. We use here the reference value $g_{\mathrm{MSSM}}=915 / 4$ instead of $g_{\mathrm{SU}(5) \times \mathrm{U}(1)}=1545 / 4$, as most of the difference is due to heavy fields, whose production is kinematically forbidden. However, as is well known, the reheating temperature does not constitute an upper bound on the effective instantaneous temperature $T$, as higher effective temperatures can be reached during the reheating process [15, 70, 81]. Using the relation

$$
T=\left(\frac{30 \rho_{\gamma}}{\pi^{2} g(T)}\right)^{1 / 4}
$$

where $\rho_{\gamma}$ denotes the instantaneous energy density of the relativistic decay products, an effective instantaneous temperature during reheating may be defined. This leads to a maximum temperature of the dilute plasma shortly after the start of inflaton decay, which may be written as

$$
T_{\max } \simeq 0.74\left(\frac{\Gamma_{s} m M_{P}^{2}}{g_{\max }}\right)^{1 / 4} \lesssim\left(\frac{915 / 4}{g_{\max }}\right)^{1 / 4}\left(3.8 \times 10^{12} \mathrm{GeV}\right),
$$


where the inequality is due to the gravitino production bound. As discussed in [15], because of the finite rate of the thermalization process, the actual maximum temperature of the Universe is in the range $T_{\max } \gtrsim T>T_{\text {reh. }}$.

The previous constraints on the decay rate of the inflaton, and the maximum temperatures during and after reheating, change if there is an intermediate matter-dominated phase between the end of reheating and the end of the radiation-dominated era. We consider this effect in Section 5.3.3.

\subsection{Supercosmology and The GUT phase transition}

The maximum temperature during reheating is typically a few orders of magnitude lower than the GUT scale. However, due to the stabilization of $\widetilde{\nu}_{H}^{c}, \widetilde{\nu}_{\bar{H}}^{c}$ at their origins during inflation, the Universe enters the reheating epoch in an $\mathrm{SU}(5) \times \mathrm{U}(1)$ symmetric state. The eventual breaking of the symmetry takes place along an $F$ - and $D$-flat direction of the potential $\tilde{\nu}_{H}^{c}=\tilde{\nu}_{\bar{H}}^{c} \equiv \Phi$ and finite-temperature corrections to the effective potential must be taken into account. As we will see, the strong coupling behaviour of SU(5) at low temperatures help drive the transition [37,82,

The leading-order running of the $\mathrm{SU}(5)$ gauge coupling is

$$
\frac{1}{\alpha_{5}(\mu)}=\frac{1}{\alpha_{\mathrm{GUT}}}-\frac{b_{5}}{2 \pi} \ln \left(\frac{M_{\mathrm{GUT}}}{\mu}\right)
$$

where $b_{5}=15-\left(n_{5}+3 n_{10}\right) / 2$ with $n_{5}\left(n_{10}\right)$ the number of $\mathbf{5}$ and $\overline{\mathbf{5}}(\mathbf{1 0}$ and $\overline{\mathbf{1 0}})$ multiplets. For the field content introduced in Section $2, n_{5}=5$ and $n_{10}=5$, and thus $b_{5}=5$. As discussed above in (10), $\alpha_{\mathrm{GUT}} \simeq 0.0374$ for $M_{\mathrm{GUT}} \simeq 1.2 \times 10^{16} \mathrm{GeV}$, and equation (82) implies that the $\mathrm{SU}(5)$ group is asymptotically free, with the coupling in the unbroken phase becoming strong at a large energy scale $\Lambda_{c} \gg m_{W}$. Naively, this scale would be associated with the condition $g_{5} \sim 1$, corresponding to $\mu \sim 2 \times 10^{8} \mathrm{GeV}$, but symmetry-breaking bilinear condensates may be formed above/below this threshold. These condensates acquire masses of order $\Lambda_{c}$ and effectively decouple from the low-energy theory. The nature of the condensates and their transformation properties can in principle be investigated using a generalization of the so-called most attractive channel (MAC) hypothesis [83,84]. Schematically, lattice calculations have indicated that the exchange potential for the fermion bilinear may be sufficiently large for condensation to occur if [85]

$$
g^{2}\left(\Lambda_{c}\right) \Delta C \equiv g^{2}\left(\Lambda_{c}\right)\left(C_{c}-C_{1}-C_{2}\right) \simeq 4, \quad \alpha_{c} \equiv \alpha\left(\Lambda_{c}\right) \simeq \frac{1}{\pi \Delta C}
$$

where $C_{c}, C_{1}$ and $C_{2}$ denote the quadratic Casimirs of the composite channel and the 
two elementary supermultiplets, respectively 18 . More specifically, it is assumed that the channel that maximizes the effective coupling (the MAC) is the one that condenses first. Then, a second MAC condensate may form in the new broken phase, further reducing the symmetry group of the model. Successive MACs continue to condense until the non-Abelian gauge group is completely broken.

The MAC spectrum for the flipped model was studied within this approach in [37], under the additional condition that supersymmetry remains unbroken during the MAC formation. We do not repeat that analysis here, but summarize the results. All in all, the total number of light states in the strong-coupling phase is expected to be at most $4\left(\mathbf{1}_{i}\right)+1(\mathbf{1})+14\left(\mathbf{1}_{4}\right)+3\left(\mathbf{1}_{j}\right)+3\left(\ell_{i}^{c}\right)=25$ where (as discussed in detail in [37]), the $\mathbf{1}_{i}$ are singlet fields arising from $\mathbf{1 0}_{i} \times \overline{\mathbf{1 0}}_{\bar{H}}$ condensation (where $i=1,2,3, H$ ), $\mathbf{1}$ is a singlet arising from $\boldsymbol{5}_{h} \times \overline{\mathbf{5}}_{\bar{h}}$ condensation, and the $\mathbf{1}_{j}$ are SU(4) singlets in the $\overline{\mathbf{5}}_{j}$ matter representations. Among these, 14 get masses $\propto \Phi$ and 11 do not, which is fewer than in [37], as the singlets $\phi_{a}$ are not included among the light states, as we assume here the presence of the bilinear couplings $\mu^{a b}$. This is to be contrasted with the $\mathrm{SU}(5) \times \mathrm{U}(1)$-symmetric phase, which has 103 light superfield degrees of freedom, and the Higgs phase, in which 62 do not acquire masses $\sim \Phi$.

As a representative example of the net Casimir coefficient $\Delta C$ in a specific MACs, we may take $\Delta C=\frac{24}{5}$ in (83), which gives $\alpha_{c} \simeq 0.0663$. Then, from Eq. (82) we have

$$
\Lambda_{c} \simeq M_{\mathrm{GUT}} \exp \left[-\frac{2 \pi}{b_{5}}\left(\frac{1}{\alpha_{\mathrm{GUT}}}-\frac{1}{\alpha_{c}}\right)\right] .
$$

Using our previous estimate that $\alpha\left(M_{\mathrm{GUT}}\right)=0.0374$, we find that strong-coupling dynamics will be important for

$$
\Lambda_{c} \simeq 4 \times 10^{-7} M_{\mathrm{GUT}} .
$$

Using also our estimate $M_{\mathrm{GUT}}=1.2 \times 10^{16} \mathrm{GeV}$, we then have $\Lambda_{c} \simeq 5 \times 10^{9} \mathrm{GeV}$. Again using (83), other MAC channels give estimates of $\Lambda_{c}$ between $10^{8}$ and $10^{14} \mathrm{GeV} 19$. As we will see in Section [5.3, if $T_{R} \gtrsim \Lambda_{c} \gtrsim \sqrt{\left|m_{\Phi}\right| M_{\mathrm{GUT}}} \sim 10^{10} \mathrm{GeV}$, the oscillation of the $\Phi$ field occurs incoherently, which minimizes the entropy release due to $\Phi$ decay. Eq. (85) suggests that it is plausible that $\Lambda_{c}$ falls into this region, within the large current uncertainties.

The difference in the number of light degrees of freedom between the symmetric, strongly-coupled and Higgs phases of the theory is crucial for the onset of the $\mathrm{SU}(5) \times$ $\mathrm{U}(1) \rightarrow \mathrm{SU}(3) \times \mathrm{SU}(2) \times \mathrm{U}(1)$ phase transition, as we now show. From the one-loop

\footnotetext{
${ }^{18}$ Larger values of $\alpha_{c}$ are estimated in the ladder approximation [86]. The differences between the various estimates of $\alpha_{c}$ stem from our incomplete understanding of strong dynamics.

${ }^{19}$ For example, $\Delta C=36 / 5$ gives $\alpha_{c}=0.0442$ and $\Lambda_{c}=6 \times 10^{-3} M_{\mathrm{GUT}}, \Delta C=18 / 5$ gives $\alpha_{c}=0.0884$ and $\Lambda_{c}=4 \times 10^{-9} M_{\mathrm{GUT}}$, and $\Delta C=15 / 4$ gives $\alpha_{c}=0.0849$ and $\Lambda_{c}=7 \times 10^{-9} M_{\mathrm{GUT}}$. See 37] for more details.
} 


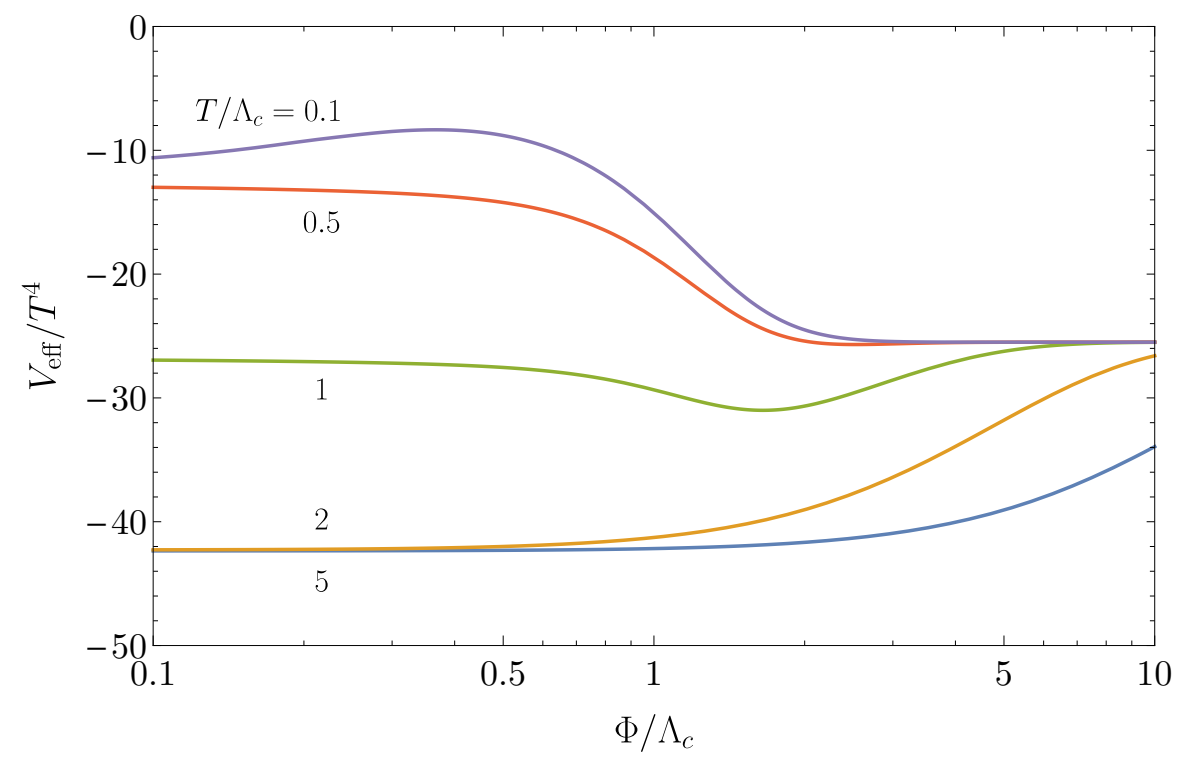

Figure 12: The evolution with temperature of the effective potential $V_{\mathrm{eff}}(\Phi, T)$ (87) in strongly-coupled $S U(5) \times U(1)$.

temperature-dependent correction to the effective potential, we have a contribution from light superfields that remain massless in the broken phases equivalent to that of an ideal ultrarelativistic gas, i.e.,

$$
V_{\text {eff, light }}=-\frac{\pi^{2} T^{4}}{90} g
$$

and the $\Phi$-independent heavy states will have negligible contributions. For the states with $\Phi$-dependent masses, there are contributions to the chiral mass-squared matrices proportional to $\left|\lambda_{4,5,6}\right|^{2} \Phi^{2}$, and to $g^{2} C_{a} \Phi^{2}$ for the vector superfields. Under the assumption that $\lambda_{4,5,6}, g_{a} C_{a} \sim \mathcal{O}(1)$ in the strong-coupling domain, we may write a phenomenological fit to the temperature-dependent effective potential of the form

$$
V_{\mathrm{eff}}(\Phi, T) \approx N_{\Phi} \frac{T^{4}}{2 \pi^{2}} \sum_{\alpha=0,1}(-1)^{\alpha} \int_{0}^{\infty} d y y^{2} \ln \left[1-(-1)^{\alpha} \exp \left(-\sqrt{y^{2}+(\Phi / T)^{2}}\right)\right]
$$

where $N_{\Phi}$ denotes the number of $\Phi$-dependent massive superfields in the corresponding regime. Fig. 12 shows the resulting shape of the effective potential as a function of $\Phi$ when $T / \Lambda_{c}=\mathcal{O}(1)$. For definiteness, we have used a smooth (logistic function) interpolation for $g$ and $N_{\Phi}$ around the strong-coupling-transition scale $\Lambda_{c}$. In the topmost curve, a barrier that might trap $\Phi$ near the origin when $T<\Lambda_{c}$ is apparent. This effect may be an artifact of the approximations that we have considered, and we expect in any case that strongcoupling and thermal effects would easily make an end run around any such barrier when $T \sim \Lambda_{c}$. 


\subsection{Entropy release}

Having verified how the phase transition takes place, we now estimate the amount of entropy it releases. In what follows, we denote the decay rate of the flat direction by $\Gamma_{\Phi}$, and the scale factor by $a$. As we will discuss below, the amount of entropy release will be dependent on whether it is possible or not for the flat direction $\Phi$ to undergo coherent oscillations after the completion of the phase transition [36].

One possibility is that reheating takes place at temperatures lower than the strongcoupling scale, $T_{\text {reh }}<\Lambda_{c}$. In this weak reheating scenario, the $\mathrm{SU}(5) \times \mathrm{U}(1)$ gauge symmetry is not restored after inflation, and the field $\Phi$ eventually reaches its low-energy minimum and reheats the Universe through the coherent decay of its oscillations. Disregarding non-renormalizable terms that could lift the flat direction, field dependence in the zero-temperature effective potential for $\Phi$ can only come from a supersymmetry-breaking term $\sim m_{\Phi}^{2} \Phi^{2}$, where $m_{\Phi}^{2}$ is assumed to be negative. The energy stored in the scalar field oscillations of $\Phi$ following the phase transition may then be simply estimated as

$$
\rho_{\Phi} \simeq\left|m_{\Phi}^{2}\right|\langle\Phi\rangle^{2}\left(\frac{a(t)}{a_{\Phi}}\right)^{-3} .
$$

where $\langle\Phi\rangle$ denotes the low-temperature vev of $\Phi$, responsible for the breaking of the GUT symmetry, and $a_{\Phi}$ denotes the size of the scale factor at the onset of $\Phi$-oscillations.

In contrast, for strong reheating, $T_{\text {reh }} \gtrsim \Lambda_{c}$, and the $\Phi$ field starts growing as the temperature falls below $\Lambda_{c}$. This growth, however, will be driven by incoherent fluctuations, in which there is a sizeable kinetic energy for the $\Phi$ field, $\dot{\Phi} \sim T^{2}$. For $\Lambda_{c}>\left(\left|m_{\Phi}\right| \Phi\right)^{1 / 2}$, the incoherent component of $\Phi$ will dominate and destroy any coherent contribution 20 . The flat direction will then redshift as radiation until its temperature decreases sufficiently to bring it to the non-relativistic regime, during which it eventually decays and reheats the Universe.

In the following subsections we determine the amount of entropy released by the decay of $\Phi$ in the weak and strong reheating scenarios, and determine their effect on the final baryon asymmetry.

\subsubsection{Weak reheating}

We consider first the weak reheating case, for which the GUT gauge symmetry is not restored after inflation, and the $\Phi$ condensate oscillates coherently about its low-energy

\footnotetext{
${ }^{20}$ Even in the presence of coherence, the condition for fast damping of the field oscillations, $\left|m_{\Phi}\right| \sim$ $H \sim \Lambda_{c}^{2} / M_{P}$, is not violated by more than an order of magnitude, implying complete damping after a few oscillations.
} 
minimum. Dependent on the magnitudes of the decay rates of the inflaton $s$ and the flat direction $\Phi$, the later can begin its oscillations and/or decay before or after the completion of reheating. It is clear that a short-lived $\Phi$, namely one which decays before the end of reheating, will not contribute significantly towards the production of entropy, which continues until the end of reheating. Therefore, to explore the potential entropy injection produced by $\Phi$ decay, we need to consider only the case in which $\Gamma_{s}>\Gamma_{\Phi}$. Let us assume first that the flat direction starts oscillations during the radiation-dominated era with $\left|m_{\Phi}\right|<\Gamma_{s}$. As the energy density of the inflaton decay products at the end of reheating is $\rho_{\gamma, \text { reh }} \sim \Gamma_{s}^{2} M_{P}^{2}[13,15]$, we can write at later times

$$
\rho_{\gamma} \sim \Gamma_{s}^{2} M_{P}^{2}\left(\frac{a(t)}{a_{\mathrm{reh}}}\right)^{-4} .
$$

With $a(t) \sim t^{1 / 2}$ during radiation domination and the Hubble parameter given by $H_{\gamma} \sim$ $\rho_{\gamma}^{1 / 2} / M_{P}$, and assuming for simplicity the instantaneous onset of oscillations and decay of $\Phi$ when $H\left(t_{\Phi}\right) \sim\left|m_{\Phi}\right|$ and $H\left(t_{d \Phi}\right) \sim \Gamma_{\Phi}$, respectively, the ratio

$$
\left.\frac{\rho_{\Phi}}{\rho_{\gamma}}\right|_{d \Phi} \sim \frac{\left|m_{\Phi}^{2}\right|\langle\Phi\rangle^{2}}{\Gamma_{s}^{2} M_{P}^{2}} \frac{a_{\Phi}^{3} a_{d \Phi}}{a_{\mathrm{reh}}^{4}} \simeq \frac{\left|m_{\Phi}\right|^{1 / 2}\langle\Phi\rangle^{2}}{\Gamma_{\Phi}^{1 / 2} M_{P}^{2}},
$$

will be smaller than one if the following constraint on the decay rate of $\Phi$ is satisfied,

$$
\frac{\Gamma_{\Phi}}{\left|m_{\Phi}\right|}>\left(\frac{\langle\Phi\rangle}{M_{P}}\right)^{4} \gtrsim 2 \times 10^{-11}
$$

for $\langle\Phi\rangle \gtrsim 5 \times 10^{15} \mathrm{GeV}$ as required by the proton lifetime (see Eq. (13)). If this occurs, a negligible amount of entropy will be released upon $\Phi$ decay. When (91) is not satisfied, the oscillations of $\Phi$ will eventually dominate the energy density of the Universe. In this case, from (88) and (89), we can compute the scale factor $a_{*}$ at $\Phi$-radiation equality as

$$
\frac{a_{*}}{a_{\Phi}} \simeq\left(\frac{M_{P} \Gamma_{s}}{\left|m_{\Phi}\right|\langle\Phi\rangle}\right)^{2}\left(\frac{a_{\mathrm{reh}}}{a_{\Phi}}\right)^{4} \simeq\left(\frac{M_{P}}{\langle\Phi\rangle}\right)^{2} .
$$

With the Hubble parameter during $\Phi$ domination given by

$$
H_{\Phi} \sim \frac{\left|m_{\Phi}\right|\langle\Phi\rangle}{M_{P}}\left(\frac{a_{\Phi}}{a}\right)^{3 / 2}
$$

and the decay of the flat direction occurring at $\Gamma_{\Phi} \sim H_{\Phi}$, we can re-calculate the ratio (90) for the flat direction-dominated case as 21

$$
\left.\frac{\rho_{\Phi}}{\rho_{\gamma}}\right|_{d \Phi}=\left(\frac{a_{d \Phi}}{a_{*}}\right)=\left(\frac{a_{d \Phi}}{a_{\Phi}}\right)\left(\frac{a_{\Phi}}{a_{*}}\right) \sim\left(\frac{\left|m_{\Phi}\right|\langle\Phi\rangle^{4}}{\Gamma_{\Phi} M_{P}^{4}}\right)^{2 / 3} .
$$

\footnotetext{
${ }^{21}$ Here we have assumed that $\rho_{\gamma}$ goes as $a^{-4}$ during the whole epoch. Generically speaking, during the coherent oscillation of $\Phi$, the radiation originating from the decay of $\Phi$ might have dominated that from inflaton decay. In this case, the Universe would experience non-adiabatic expansion and $\rho_{\gamma}$ goes as $a^{-3 / 2}$ [87]. However, it turns out that the non-adiabatic expansion occurs only at a very low temperature because of the small decay rate of $\Phi$ (see below), and thus this modification would be negligible in our scenario.
} 
which is $>1$, consistent with the violation of the condition (911).

Let us now assume that the oscillation of $\Phi$ starts during reheating, $\left|m_{\Phi}\right|>\Gamma_{s}$. In this case we can interpolate between the start of oscillations of the flat direction and the end of reheating as

$$
\left(\frac{a_{\mathrm{reh}}}{a_{\Phi}}\right) \sim\left(\frac{\left|m_{\Phi}\right|}{\Gamma_{s}}\right)^{2 / 3} .
$$

If the Universe is dominated by radiation by the time of the decay of $\Phi$, we will have

$$
\left.\frac{\rho_{\Phi}}{\rho_{\gamma}}\right|_{d \Phi} \sim \frac{\left|m_{\Phi}^{2}\right|\langle\Phi\rangle^{2}}{\Gamma_{s}^{2} M_{P}^{2}}\left(\frac{a_{\Phi}}{a_{\mathrm{reh}}}\right)^{3}\left(\frac{a_{d \Phi}}{a_{\mathrm{reh}}}\right) \simeq \frac{\Gamma_{s}^{1 / 2}\langle\Phi\rangle^{2}}{\Gamma_{\Phi}^{1 / 2} M_{P}^{2}} .
$$

Thus, no significant amount of entropy will be released if the following constraint on the decay rate of $\Phi$ is satisfied,

$$
\frac{\Gamma_{\Phi}}{\Gamma_{s}}>\left(\frac{\langle\Phi\rangle}{M_{P}}\right)^{4} \gtrsim 2 \times 10^{-11} .
$$

Conversely, if the previous relation is violated, the flat direction oscillations will dominate the energy density until their decay. In this scenario, we can write

$$
\left.\frac{\rho_{\Phi}}{\rho_{\gamma}}\right|_{d \Phi}=\left(\frac{a_{d \Phi}}{a_{*}}\right)=\left(\frac{a_{d \Phi}}{a_{\Phi}}\right)\left(\frac{a_{\Phi}}{a_{*}}\right) \sim\left(\frac{\Gamma_{s}\langle\Phi\rangle^{4}}{\Gamma_{\Phi} M_{P}^{4}}\right)^{2 / 3}>1 .
$$

With the entropy density in radiation given by $s_{\gamma}=4 / 3\left(g_{\mathrm{reh}} \pi^{2} / 30\right)^{1 / 4} \rho_{\gamma}^{3 / 4}$, and a similar expression for the entropy density produced from $\Phi$ decays, we can summarize the amount of entropy released in both scenarios as

$$
\left.\Delta \equiv \frac{s_{\Phi}}{s_{\gamma}}\right|_{d \Phi} \sim\left(\frac{g_{d \Phi}}{g_{\mathrm{reh}}}\right)^{1 / 4}\left(\frac{\min \left[\left|m_{\Phi}\right|, \Gamma_{s}\right]\langle\Phi\rangle^{4}}{\Gamma_{\Phi} M_{P}^{4}}\right)^{1 / 2} .
$$

As was discussed in [37, the decay rate of the flat direction occurs via the effective $D$-term diagrams shown in Fig. 13, which lead to the decay rate

$$
\Gamma_{\Phi} \simeq \frac{9 \lambda_{1,2,3,7}^{4}}{2048 \pi^{5}}\left(\frac{\left|m_{\Phi}\right| m_{F, \bar{f}, \ell^{c}, \tilde{\phi}_{a}}^{2}}{\langle\Phi\rangle^{2}}\right) .
$$

The gravitino production constraint implies that $\Gamma_{s} \lesssim 900 \mathrm{GeV}$ (see (78)) and, if we assume that the effective mass in the flat direction is heavier than the weak scale, the entropy ratio (99) evaluates to

$$
\begin{aligned}
& \Delta \simeq 1.5 \times 10^{8} \lambda_{1,2,3,7}^{-2}\left(\frac{g_{d \Phi}}{g_{\mathrm{reh}}}\right)^{1 / 4}\left(\frac{\langle\Phi\rangle}{5 \times 10^{15} \mathrm{GeV}}\right)^{3}\left(\frac{(10 \mathrm{TeV})^{3}}{m_{F, \bar{f}, \ell^{c}, \tilde{\phi}_{a}}^{2}\left|m_{\Phi}\right|}\right)^{1 / 2}\left(\frac{\Gamma_{s}}{900 \mathrm{GeV}}\right)^{1 / 2} \simeq \\
& 6.2 \times 10^{7} \lambda_{1,2,3,7}^{-2}\left(\frac{g_{d \Phi}}{g_{\mathrm{reh}}}\right)^{1 / 4}\left(\frac{\langle\Phi\rangle}{5 \times 10^{15} \mathrm{GeV}}\right)^{3}\left(\frac{(10 \mathrm{TeV})^{3}}{m_{F, \bar{f}, \ell^{c}, \tilde{\phi}_{a}}^{2}\left|m_{\Phi}\right|}\right)^{1 / 2}\left(\frac{y}{10^{-5}}\right)\left(\frac{m}{3 \times 10^{13} \mathrm{GeV}}\right)^{1 / 2} .
\end{aligned}
$$



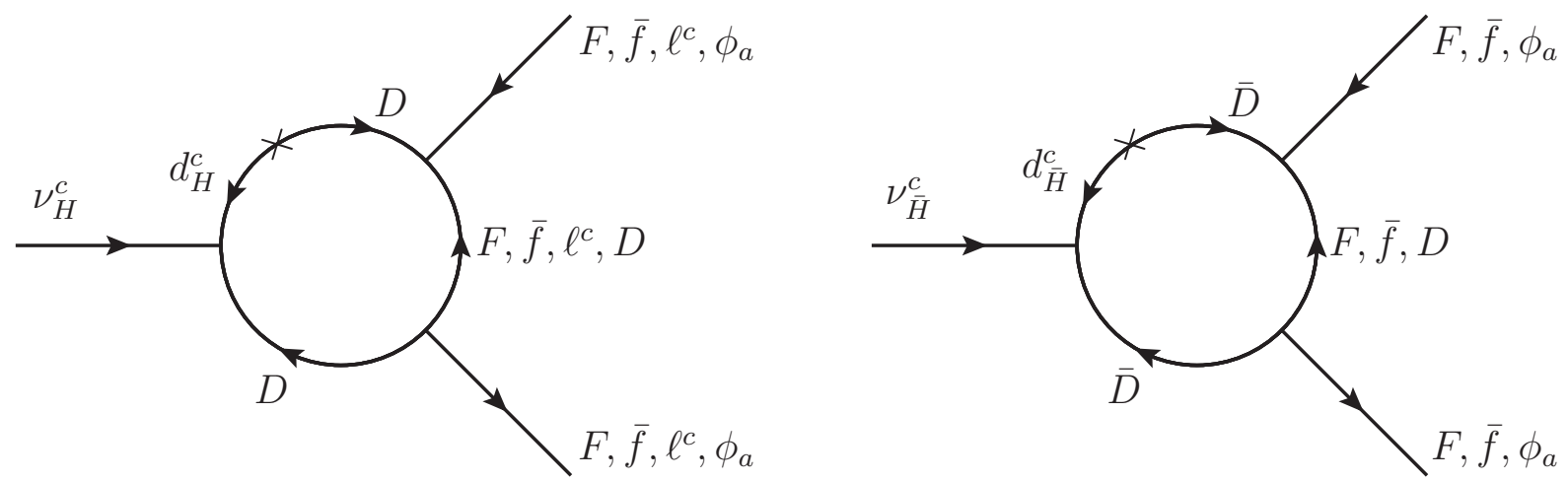

Figure 13: Diagrams contributing to a D-term generating the decay rate (100) for the $\Phi$ field in the flipped $S U(5) \times U(1)$ GUT model.

A large release of entropy could be problematic if it overly dilutes the baryon asymmetry. But we should bear in mind that 1) the estimate in (101) is proportional to $y$ and so may be significantly smaller if $y$ is small; 2) the matter-antimatter asymmetry may be quite large initially, e.g., if generated via the Affleck-Dine mechanism [88, in which case some dilution could be acceptable or even welcome. Such dilution can also relax the gravitino over-production problem as we see in Section 5.3.3.

\subsubsection{Strong reheating}

When the temperature of the relativistic plasma following reheating is $T_{\max }>T_{\text {reh }} \sim \Lambda_{c}$, the energy density of the flat direction $\Phi$ will be dominated by incoherent fluctuations of energy $\mathcal{O}\left(\Lambda_{c}\right)$ as the phase transition to the broken symmetry phase takes place. As was discussed earlier, these fluctuations will erase the coherent component of $\Phi$. Eventually, the interactions that lifted the energy of $\Phi$ to the plasma temperature will cease to maintain it in equilibrium, and the flat direction will decouple at $T_{\mathrm{dec}} \lesssim \Lambda_{c}$, maintaining a profile that evolves as $T_{\Phi}=T_{\mathrm{dec}}\left(a_{\mathrm{dec}} / a\right)=T\left(g(T) / g_{\mathrm{dec}}\right)^{1 / 3}$, where $T$ is the temperature of the radiation background. At a later time, this temperature will fall below $\left|m_{\Phi}\right|$, $\Phi$ will become non-relativistic, and the Universe will be matter-dominated until the decay of $\Phi$ at $T_{d \Phi}$. Equating the Hubble rate $H=\sqrt{\rho_{\Phi} / 3 M_{P}^{2}}$, with $\rho_{\Phi}=\zeta(3)\left|m_{\Phi}\right| T_{\Phi}^{3} / \pi^{2}$, to the decay rate (100) one obtains

$$
T_{d \Phi} \simeq 2 \times 10^{-3} \lambda_{1,2,3,7}^{8 / 3}\left(\frac{\left|m_{\Phi}\right| m_{F, \bar{f}, \ell^{c}, \tilde{\phi}_{a}}^{4} M_{P}^{2}}{\langle\Phi\rangle^{4}}\right)^{1 / 3}
$$


Upon the decay of $\Phi$, the Universe will be once again dominated by radiation, with a temperature now given by

$$
T_{\mathrm{reh}}^{\prime}=\left(\frac{40}{g_{d \Phi} \pi^{2}}\right)^{1 / 4}\left(\Gamma_{\Phi} M_{P}\right)^{1 / 2} \simeq 5 \times 10^{-3} g_{d \Phi}^{-1 / 4} \lambda_{1,2,3,7}^{2}\left(\frac{\left|m_{\Phi}\right| m_{F, \bar{f}, \ell^{c}, \tilde{\phi}_{a}}^{2} M_{P}}{\langle\Phi\rangle^{2}}\right)^{1 / 2}
$$

where we have neglected the delay arising from the conversion of the heavy supersymmetric decay products into the truly relativistic Standard Model particles. For $\langle\Phi\rangle \simeq 5 \times 10^{15} \mathrm{GeV}$, $\lambda_{1,2,3,7} \sim 1$ and $m_{\Phi, F, \bar{f}, \ell^{c}, \tilde{\phi}_{a}} \gtrsim 10 \mathrm{TeV}$, this temperature is $T_{\text {reh }}^{\prime} \gtrsim 1 \mathrm{MeV}$, remarkably around what is needed to re-start nucleosynthesis. The amount of entropy released by the decay of $\Phi$ can therefore be estimated as

$$
\begin{aligned}
\Delta & =\frac{g_{d \Phi} T_{\mathrm{reh}}^{\prime 3}}{\left.\left(g(T) T^{3}\right)\right|_{d \Phi}}=\frac{g_{d \Phi} T_{\mathrm{reh}}^{\prime 3}}{g_{\mathrm{dec}} T_{d \Phi}^{3}} \\
& \simeq 8 \times 10^{3} \lambda_{1,2,3,7}^{-2}\left(\frac{g_{d \Phi}}{43 / 4}\right)^{1 / 4}\left(\frac{915 / 4}{g_{\mathrm{dec}}}\right)\left(\frac{\langle\Phi\rangle}{5 \times 10^{15} \mathrm{GeV}}\right)\left(\frac{10 \mathrm{TeV}}{m_{F, \bar{f}, \ell^{c}, \tilde{\phi}_{a}}^{2} /\left|m_{\Phi}\right|}\right)^{1 / 2} .
\end{aligned}
$$

Therefore, in the strong reheating scenario the entropy dilution is reduced by $\mathcal{O}\left(10^{4}\right)$ with respect to weak reheating, providing more leeway for the initial asymmetry-generating mechanism.

\subsubsection{Entropy production and the gravitino bound on reheating}

A late injection of entropy would dilute any previously produced relics, such as gravitinos or their decay products. In particular, the gravitino yield $Y_{3 / 2} \equiv n_{3 / 2} / n_{\gamma}$ would be reduced by a factor of $\Delta^{-1}$. Since in the absence of a secondary matter-dominated era driven by $\Phi$, the yield at late times is given approximately by [15]

$$
Y_{3 / 2}(T) \simeq 0.0036\left(1+0.56 \frac{m_{1 / 2}^{2}}{m_{3 / 2}^{2}}\right)\left(\frac{\Gamma_{s}}{M_{P}}\right)^{1 / 2}
$$

the extra dilution would weaken the decay rate constraint imposed by the LSP relic density by a factor of $\Delta^{2}$, thus allowing a higher reheat temperature by a factor of $\Delta$. More specifically, (78) would now become

$$
|y|<2.7 \times 10^{-5} \Delta\left(1+0.56 \frac{m_{1 / 2}^{2}}{m_{3 / 2}^{2}}\right)^{-1}\left(\frac{100 \mathrm{GeV}}{m_{\mathrm{LSP}}}\right),
$$

and $T_{\text {reh }} \lesssim \Delta\left(10^{10} \mathrm{GeV}\right)$. Thus, the strong reheating condition $T_{\text {reh }} \gtrsim \Lambda_{c}$ would automatically be allowed by the late decay of the flaton $\Phi$. 
An additional effect of the entropy increase (101) or (105) would be a shift in the number of $e$-folds of inflation after the pivot scale $k_{*}$ crosses the horizon, due to the nonstandard thermal history [89,90]. In the slow-roll approximation, the number of $e$-folds to the end of inflation can be expressed as [21,49, 91]

$$
N_{*}=\ln \left(\frac{\rho_{\mathrm{reh}}^{1 / 4} a_{\mathrm{reh}}}{\sqrt{3} a_{0} H_{0}}\right)-\ln \left(\frac{k_{*}}{a_{0} H_{0}}\right)+\frac{1}{4} \ln \left(\frac{V_{*}^{2}}{M_{P}^{4} \rho_{\text {end }}}\right)+\frac{1-3 w_{\text {int }}}{12\left(1+w_{\text {int }}\right)} \ln \left(\frac{\rho_{\text {reh }}}{\rho_{\text {end }}}\right),
$$

where $a_{0}$ and $H_{0}$ are the present cosmological scale factor and Hubble expansion rate, respectively, $V_{*}$ is the inflationary energy density at the reference scale, $\rho_{\text {end }}$ and $\rho_{\text {reh }}$ are the energy densities at the end of inflation and after reheating, and $w_{\text {int }}$ is the $e$-fold average of the equation-of-state parameter during the thermalization epoch. For the standard thermal history (STH), the first term evaluates to $66.9-\frac{1}{12} \ln g_{\mathrm{reh}}$, as entropy is assumed to be conserved after reheating. In our case of an intermediate matter-dominated era, it can be rewritten as

$$
\ln \left(\frac{\rho_{\mathrm{reh}}^{1 / 4} a_{\mathrm{reh}}}{\sqrt{3} a_{0} H_{0}}\right)=\ln \left(\frac{\rho_{d \Phi}^{1 / 4} a_{d \Phi}}{\sqrt{3} a_{0} H_{0}}\right)+\ln \left(\frac{\rho_{\mathrm{reh}}^{1 / 4} a_{\mathrm{reh}}}{\rho_{d \Phi}^{1 / 4} a_{d \Phi}}\right),
$$

where now the first term of (109) can be evaluated assuming entropy conservation, and the second term is directly related to the dilution factor $\Delta$. We obtain for the number of $e$-folds the following expression,

$$
N_{*}=N_{*}^{\mathrm{STH}}-\frac{1}{3} \ln \Delta .
$$

At first sight, the reduction of $N_{*}$ due to the late entropy injection might appear to put Starobinsky-like inflation under stress, as the Planck data disfavors $N_{*} \lesssim 50$ (44) at the $68 \%(95 \%)$ CL. However, the physical range for $N_{*}$ depends on the underlying particle model, since the last term in (108) depends implicitly on the decay rate of the inflaton. For Starobinsky-like inflation, it can be evaluated in the perturbative regime as [13]

$$
\frac{1-3 w_{\mathrm{int}}}{12\left(1+w_{\mathrm{int}}\right)} \ln \left(\frac{\rho_{\mathrm{reh}}}{\rho_{\mathrm{end}}}\right) \simeq \frac{1-3 w_{\mathrm{int}}}{12\left(1+w_{\mathrm{int}}\right)}\left(2 \ln \left(\frac{\Gamma_{s}}{m}\right)+\text { const. }\right)
$$

where $w_{\text {int }} \simeq 0.782 / \ln \left(2 m / \Gamma_{s}\right)$. In the STH case, the gravitino upper bound for $\Gamma_{s}$ constrains $N_{*}$ to be less than $N_{*}^{\max } \simeq 53.3$. However, in the case of intermediate $\Phi$-domination, this maximum value is reduced due to the modified $e$-fold expression (110), and also increased because of the weakened gravitino bound (107). These two effects combine to give

$$
\Delta N_{*}^{\max } \simeq-4 \times 10^{-3} \ln \Delta .
$$

Therefore, the favored range for $N_{*}$ is practically unchanged for strong reheating when one accounts for the increased decay rate limit. In the weak reheating regime, the maximum 
value for $N_{*}$ cannot be reached within perturbation theory, as for $|y| \lesssim 1$ the number of $e$-folds is limited to $N_{*} \lesssim 49$ with $\Delta \simeq 5 \times 10^{8}$, the value of the entropy factor obtained by taking $\min \left[\left|m_{\Phi}\right|, \Gamma_{s}\right]=\left|m_{\Phi}\right|$ in (99). Hence, for weak reheating, the inflationary predictions lie outside the $1 \sigma$ Planck bounds.

\subsubsection{Baryon asymmetry}

Finally, let us investigate the generation of the baryon asymmetry in this class of models. In Scenario (A), as we noted earlier, at the tree level the inflaton decays primarily to Higgs bosons and Higgsinos, and there is no decay to neutrinos. At one-loop, through the exchange of a heavy right-handed neutrino, there is the possibility of a lepton-numberviolating decay to two neutrinos. However, in that case, in order to obtain a net lepton asymmetry one must consider the interference between one-loop diagrams and their twoloop corrections, greatly suppressing the final lepton asymmetry.

However, in the case of strong reheating in Scenario (A), it may be possible to produce thermally the right-handed neutrinos, though this is possible only if the reheating temperature is comparable to the right-handed neutrino mass. We recall however, that in Scenario (A) the right-handed neutrino mass is of order $\lambda_{6}^{i j}\left\langle\tilde{\nu}_{\bar{H}}^{c}\right\rangle\left(\right.$ for $\left.\tilde{\nu}_{\bar{H}}^{c}\right\rangle \sim \mu^{i j}$ ) and, from (79), the reheating temperature is $T_{R} \lesssim 8.7 \times 10^{14}\left|\lambda_{7}^{0}\right| \mathrm{GeV} \lesssim 8.7 \times 10^{9} \Delta \mathrm{GeV}$. Thus we would require

$$
\lambda_{6}^{i j} \lesssim 1.7 \times 10^{-6}\left(\frac{5 \times 10^{15} \mathrm{GeV}}{\left\langle\tilde{\nu}_{\bar{H}}^{c}\right\rangle}\right) \Delta .
$$

Taking into account the entropy production factor in (104), this is a viable path towards producing the final baryon asymmetry.

Generating the baryon asymmetry is more straightforward in Scenario (B), in which the inflaton decays to $L h$ in much the same way that right-handed neutrinos decay in standard out-of-equilibrium leptogenesis models [55]. At the end of inflation, the lepton and baryon asymmetries can be related directly to the reheat temperature, $T_{R}$, by [5,61,92,94]

$$
\frac{n_{B}}{s} \sim \frac{n_{L}}{s} \sim \frac{\epsilon}{\Delta} f \frac{n_{s}}{T_{R}^{3}} \sim \frac{\epsilon}{\Delta} f \frac{T_{R}}{m},
$$

where $n_{s}$ is the number density of inflatons at the time of their decay, and $f$ is the branching fraction into $L h,(f \sim 1$ for Scenario (B)). The amount of $\mathrm{C}$ and $\mathrm{CP}$ is given by [7,95]

$$
\epsilon \simeq-\frac{3}{4 \pi} \frac{1}{\left(U_{\nu^{c}}^{\dagger}\left(\lambda_{2}^{D}\right)^{2} U_{\nu^{c}}\right)_{11}} \sum_{i=2,3} \operatorname{Im}\left[\left(U_{\nu^{c}}^{\dagger}\left(\lambda_{2}^{D}\right)^{2} U_{\nu^{c}}\right)_{i 1}^{2}\right] \frac{m}{M_{i}},
$$

where $U_{\nu^{c}}$ is a mixing matrix associated with the diagonalization of $\tilde{\nu}_{i}^{c}$ and $S$ in the basis

where $\lambda_{2}$ is diagonalized to $\lambda_{2}^{D}$ [7], and we assume for simplicity that the second and third 
generation heavy neutrino states (two states for each generation) have similar masses $M_{2}$ and $M_{3}$, and $m \ll M_{i}$. Generically, we expect $\left(U_{\nu^{c}}^{\dagger}\left(\lambda_{2}^{D}\right)^{2} U_{\nu^{c}}\right)_{11} \sim\left(\lambda_{2}^{D}\right)_{33}^{2} \sim m_{t} /\left\langle\bar{h}^{0}\right\rangle \sim 1$ is the largest entry in $\left(\lambda_{2}^{D}\right)^{2}$. This gives

$$
|\epsilon| \sim 7 \times 10^{-3} \times\left(\frac{m}{3 \times 10^{13} \mathrm{GeV}}\right)\left(\frac{M_{i}}{10^{15} \mathrm{GeV}}\right)^{-1} \times \delta
$$

where $\delta$ denotes an $\mathcal{O}(1)$ factor that depends on the amount of $\mathrm{CP}$ violation in the matrix $U_{\nu^{c}}^{\dagger}\left(\lambda_{2}^{D}\right)^{2} U_{\nu^{c}}$. From Eq. (79)

$$
\begin{aligned}
T_{R} & \approx 0.07|y|\left(\frac{915 / 4}{g_{\mathrm{reh}}}\right)^{1 / 4}\left(m M_{P}\right)^{1 / 2} \\
& \approx 6 \times 10^{14}|y|\left(\frac{915 / 4}{g_{\mathrm{reh}}}\right)^{1 / 4}\left(\frac{m}{3 \times 10^{13} \mathrm{GeV}}\right)^{1 / 2}
\end{aligned}
$$

we have

$$
\frac{n_{B}}{s} \sim 20 \frac{\epsilon}{\Delta} f|y|\left(\frac{915 / 4}{g_{\text {reh }}}\right)^{1 / 4}\left(\frac{3 \times 10^{13} \mathrm{GeV}}{m}\right)^{1 / 2} .
$$

In the weak reheating scenario, the entropy dilution factor is given by (101), and leads to

$$
\frac{n_{B}}{s} \sim 7.3 \times 10^{-12} \epsilon f \lambda_{1,2,3,7}^{2}\left(\frac{43 / 4}{g_{d \Phi}}\right)^{1 / 4}\left(\frac{5 \times 10^{15} \mathrm{GeV}}{\langle\Phi\rangle}\right)^{3}\left(\frac{m_{F, \bar{f}_{,}, \tilde{\phi}_{a}}^{2}\left|m_{\Phi}\right|}{(10 \mathrm{TeV})^{3}}\right)^{1 / 2}\left(\frac{3 \times 10^{13} \mathrm{GeV}}{m}\right),
$$

from which it is clear that the enormous amount of dilution will lead to an insufficient asymmetry. Note that this estimate is now independent of the inflaton coupling $y$.

However, in the strong reheating regime, (105) implies that

$$
\begin{aligned}
\frac{n_{B}}{s} \simeq 2.7 \times 10^{-8} \epsilon f \lambda_{1,2,3,7}^{2}\left(\frac{43 / 4}{g_{d \Phi}}\right)^{1 / 4}\left(\frac{915 / 4}{g_{\mathrm{reh}}}\right)^{1 / 4}\left(\frac{g_{\mathrm{dec}}}{915 / 4}\right)\left(\frac{y}{10^{-5}}\right) \\
\times\left(\frac{5 \times 10^{15} \mathrm{GeV}}{\langle\Phi\rangle}\right)\left(\frac{m_{F, \bar{f}, \ell^{c}, \tilde{\phi}_{a}}^{2} /\left|m_{\Phi}\right|}{10 \mathrm{TeV}}\right)^{1 / 2}\left(\frac{m}{3 \times 10^{13} \mathrm{GeV}}\right)^{-1 / 2} .
\end{aligned}
$$

Substituting the expression (116) for $\epsilon$ and taking $M_{2,3} \sim \lambda_{6}^{2}\langle\Phi\rangle^{2} / \mu \sim \lambda_{6}^{2}\langle\Phi\rangle$ from (59) with $\langle\Phi\rangle \sim \mu$, we have

$$
\begin{aligned}
\frac{n_{B}}{s} \simeq 3.8 \times & 10^{-11} \delta f \lambda_{1,2,3,7}^{2} \lambda_{6}^{-2}\left(\frac{43 / 4}{g_{d \Phi}}\right)^{1 / 4}\left(\frac{915 / 4}{g_{\mathrm{reh}}}\right)^{1 / 4}\left(\frac{g_{\mathrm{dec}}}{915 / 4}\right)\left(\frac{y}{10^{-5}}\right) \\
& \times\left(\frac{5 \times 10^{15} \mathrm{GeV}}{\langle\Phi\rangle}\right)^{2}\left(\frac{m_{F, \bar{f}, \ell^{c}, \tilde{\phi}_{a} /\left|m_{\Phi}\right|}^{2}}{10 \mathrm{TeV}}\right)^{1 / 2}\left(\frac{m}{3 \times 10^{13} \mathrm{GeV}}\right)^{1 / 2} .
\end{aligned}
$$

Thus, if the product $\delta f \lambda_{1,2,3,7}^{2} / \lambda_{6}^{2}$ is of order 2.2, we obtain the correct baryon asymmetry. Moreover, this estimate for the asymmetry increases significantly if the weakened 
gravitino production bound (107) is saturated, in which case we would have $n_{B} / s \simeq$ $8.4 \times 10^{-7} \delta f \lambda_{1,2,3,7}^{2} / \lambda_{6}^{2}$.

Finally, we note that previously we had argued against the strong reheating case, on

the basis of a potential overdensity of flatinos, $\tilde{\Phi}$ [31]. However, there it was presumed that the only sources for the flatino mass were radiative [32, making it likely that the flatino was a long-lived LSP. However, as we have argued earlier, the flaton and flatino may receive significant mass contributions from either a GM term or a higher-dimensional superpotential term needed to lift the flat direction (or both). In such a case, we would not expect the flatino to be the LSP. The thermal LSP density may be less than the observed cold dark matter if there is significant entropy production, but the correct non-thermal density from gravitino decays could be obtained when the reheating bound is saturated.

\section{Summary and Discussion}

We have discussed in this paper the scope for constructing models of cosmological inflation based on a flipped SU(5) $\times \mathrm{U}(1)$ GUT model within the framework of no-scale supergravity. These two model ingredients are each attractive in their own rights, since flipped $\mathrm{SU}(5) \times \mathrm{U}(1)$ avoids the problem of proton stability that plagues many GUT models by incorporating a minimal and elegant missing-partner mechanism, and no-scale supergravity avoids the cosmological issues of generic supergravity models by ensuring a positive semi-definite effective potential with asymptotically-flat directions that are suitable for accommodating Starobinsky-like inflation. Moreover, both flipped $\mathrm{SU}(5) \times \mathrm{U}(1)$ and no-scale supergravity emerge naturally in models of string compactification.

Within this no-scale flipped $\mathrm{SU}(5) \times \mathrm{U}(1)$ framework, we have focused on realizations of inflation via a superpotential resembling (14), which can yield predictions for the CMB observables $\left(n_{s}, r\right)$ that resemble those of the Starobinsky model. The minimal flipped $\mathrm{SU}(5) \times \mathrm{U}(1)$ model contains 4 singlet fields that mix, in general, and we have studied the circumstances under which one of these could be the inflaton field. Generically, one may consider a scenario in which the inflaton eigenstate is hierarchically lighter than the other singlet mass eigenstates, or a scenario in which there is no such mass hierarchy. In both scenarios, we have studied the constraints on the couplings of the model for it to lead to Starobinsky-like predictions. Typically, we find predictions for the tensor-to-scalar ratio $r$ that are within a factor $\mathcal{O}(2)$ of the Starobinsky prediction, but the predictions for $n_{s}$ are much more sensitive to the model parameters, as seen in Fig. 4, and measurements of $n_{s}$ provide the tightest $\mathrm{CMB}$ constraints on them, as seen in Fig. 11.

It is important, when evaluating the no-scale flipped $\mathrm{SU}(5) \times \mathrm{U}(1)$ predictions, to take into account the cosmological evolutions of all the Standard Model singlet fields, including 
the $\tilde{\nu}^{c}$ components of the $\mathbf{1 0}$ representations $F$ of matter fields, and their analogues in the $\mathbf{1 0}+\overline{\mathbf{1 0}}$ Higgs representations, as seen in Figs. 6 to 10 . These effects were taken into account numerically in deriving the model parameter constraints shown in Fig. 11, As we have discussed, the upper limits on model parameters could be respected naturally by postulating a symmetry argument for 'segregation' between the inflaton and the other singlet fields.

Neutrino masses and mixing provide another relevant set of constraints on the noscale flipped $\mathrm{SU}(5) \times \mathrm{U}(1)$ framework, which depend whether the inflaton decouples from the neutrino sector. If it does, the inflationary implications for the neutrino mass matrix are not important, whereas if the inflaton does not decouple there are interesting model indications in favour of a Normal Hierarchy of the light neutrino masses, with predictions for the masses of the eigenstates, see (77).

We have also addressed the evolution of the Universe after inflation, discussing the post-inflationary reheating, which imposes a constraint on the inflaton decay coupling via the upper limit on the density of supersymmetric relic particles produced by the decays of gravitinos. We have also discussed the GUT phase transition, building upon a previous MAC analysis of the breaking pattern of the flipped $\mathrm{SU}(5) \times \mathrm{U}(1)$ gauge group. A final set of issues that we have studied in this paper was the amount of entropy release and the baryon asymmetry. This would have diluted the baryon asymmetry, and may be substantial in weak reheating scenarios, see (101), providing potentially an important constraint on the couplings responsible for the decays of the singlet fields in our model 22 . The entropy release would be considerably smaller in strong reheating scenarios, leading to a smaller dilution of the initial matter-antimatter asymmetry and facilitating the possibility that it was generated in the decays of heavy neutrinos [55].

The overall conclusion of our work is that the ambitious no-scale flipped $\mathrm{SU}(5) \times \mathrm{U}(1)$ framework is capable of satisfying the many different types of constraints ranging from CMB measurements to neutrino masses, the dark matter density and the generation of the cosmological baryon asymmetry. The particular line we have followed is based on superpotentials resembling (14), and it should be emphasized that this is not the only option for obtaining successful Starobinsky-like predictions for the CMB observables. Nevertheless, the consistency of our framework with the available constraints, coupled with the facts that both no-scale supergravity and flipped $\mathrm{SU}(5) \times \mathrm{U}(1)$ emerge naturally in models of string compactification, suggests that it may provide a good avenue for linking a wide range of particle and cosmological phenomenology to an underlying string model.

\footnotetext{
${ }^{22}$ On the other hand, the cosmological baryon asymmetry might have been generated in a different way, e.g., via the Affleck-Dine mechanism.
} 


\section{Acknowledgements}

The work of J.E. was supported in part by the UK STFC via the research grant ST/J002798/1. The work of D.V.N. was supported in part by the DOE grant DE-FG02-13ER42020 and in part by the Alexander S. Onassis Public Benefit Foundation. The work of K.A.O. was supported in part by DOE grant de-sc0011842 at the University of Minnesota. The work of N.N. was supported by the Grant-in-Aid for Scientific Research (No.17K14270).

\section{References}

[1] S. M. Barr, Phys. Lett. 112B (1982) 219; S. M. Barr, Phys. Rev. D 40, 2457 (1989).

[2] J. P. Derendinger, J. E. Kim and D. V. Nanopoulos, Phys. Lett. 139B (1984) 170.

[3] I. Antoniadis, J. R. Ellis, J. S. Hagelin and D. V. Nanopoulos, Phys. Lett. B 194 (1987) 231.

[4] I. Antoniadis, J. R. Ellis, J. S. Hagelin and D. V. Nanopoulos, Phys. Lett. B 205 (1988) 459; Phys. Lett. B 208 (1988) 209 Addendum: [Phys. Lett. B 213 (1988) 562]; Phys. Lett. B 231 (1989) 65.

[5] J. R. Ellis, J. L. Lopez and D. V. Nanopoulos, Phys. Lett. B 292, 189 (1992) [hep-ph/9207237].

[6] J. R. Ellis, D. V. Nanopoulos and K. A. Olive, Phys. Lett. B 300, 121 (1993) hep-ph/9211325.

[7] J. R. Ellis, J. L. Lopez, D. V. Nanopoulos and K. A. Olive, Phys. Lett. B 308, 70 (1993) hep-ph/9303307.

[8] B. A. Campbell, J. R. Ellis, J. S. Hagelin, D. V. Nanopoulos and R. Ticciati, Phys. Lett. B 198, 200 (1987); J. L. Lopez, D. V. Nanopoulos and K. j. Yuan, Nucl. Phys. B 399, 654 (1993) hep-th/9203025.

[9] J. Ellis, D. V. Nanopoulos and K. A. Olive, Phys. Rev. Lett. 111 (2013) 111301 arXiv:1305.1247 [hep-th]].

[10] J. Ellis, D. V. Nanopoulos and K. A. Olive, JCAP 1310 (2013) 009 arXiv:1307.3537 [hep-th]].

[11] J. Ellis, D. V. Nanopoulos and K. A. Olive, Phys. Rev. D 89, 043502 (2014) arXiv:1310.4770 [hep-ph]]. 
[12] J. Ellis, M. A. G. Garcia, D. V. Nanopoulos and K. A. Olive, JCAP 1510, 003 (2015) arXiv:1503.08867 [hep-ph]].

[13] J. Ellis, M. A. G. Garcia, D. V. Nanopoulos and K. A. Olive, JCAP 1507, 050 (2015) arXiv:1505.06986 [hep-ph]].

[14] J. Ellis, M. A. G. Garcia, D. V. Nanopoulos and K. A. Olive, Class. Quant. Grav. 33, no. 9, 094001 (2016) arXiv:1507.02308 [hep-ph]].

[15] J. Ellis, M. A. G. Garcia, D. V. Nanopoulos, K. A. Olive and M. Peloso, JCAP 1603, 008 (2016) [arXiv:1512.05701 [astro-ph.CO]].

[16] E. Cremmer, S. Ferrara, C. Kounnas and D. V. Nanopoulos, Phys. Lett. B 133 (1983) 61; J. R. Ellis, A. B. Lahanas, D. V. Nanopoulos and K. Tamvakis, Phys. Lett. B 134 (1984) 429.

[17] A. B. Lahanas and D. V. Nanopoulos, Phys. Rept. 145 (1987) 1.

[18] A. A. Starobinsky, Phys. Lett. B 91, 99 (1980).

[19] V. F. Mukhanov and G. V. Chibisov, JETP Lett. 33, 532 (1981) [Pisma Zh. Eksp. Teor. Fiz. 33, 549 (1981)].

[20] A. A. Starobinsky, Sov. Astron. Lett. 9, 302 (1983).

[21] P. A. R. Ade et al. [Planck Collaboration], Astron. Astrophys. 594, A13 (2016) arXiv:1502.01589 [astro-ph.CO]]. P. A. R. Ade et al. [Planck Collaboration], Astron. Astrophys. 594, A20 (2016) [arXiv:1502.02114 [astro-ph.CO]].

[22] J. Ellis, M. A. G. Garcia, N. Nagata, D. V. Nanopoulos and K. A. Olive, JCAP 1611 (2016) no.11, 018 arXiv:1609.05849 [hep-ph]].

[23] S. A. Abel, Phys. Lett. B 234, 113 (1990).

[24] I. Antoniadis, J. Rizos and K. Tamvakis, Phys. Lett. B 279, 281 (1992).

[25] G. K. Leontaris and J. D. Vergados, Phys. Lett. B 305, 242 (1993) hep-ph/9301291.

[26] J. Rizos and K. Tamvakis, Phys. Lett. B 685, 67 (2010) arXiv:0912.3997 [hep-ph]].

[27] J. R. Ellis, J. L. Lopez and D. V. Nanopoulos, Phys. Lett. B 252, 53 (1990).

[28] G. K. Leontaris and K. Tamvakis, Phys. Lett. B 260, 333 (1991).

[29] T. Li, D. V. Nanopoulos and J. W. Walker, Nucl. Phys. B 846, 43 (2011) arXiv:1003.2570 [hep-ph]]. 
[30] T. Li, D. V. Nanopoulos and J. W. Walker, Phys. Lett. B 693, 580 (2010) arXiv:0910.0860 [hep-ph]]; T. Li, J. A. Maxin, D. V. Nanopoulos and J. W. Walker, Eur. Phys. J. C 72, 2246 (2012) arXiv:1208.1999 [hep-ph]].

[31] J. R. Ellis, J. S. Hagelin, S. Kelley, D. V. Nanopoulos and K. A. Olive, Phys. Lett. B 209, 283 (1988).

[32] M. Drees and X. Tata, Phys. Lett. B 206, 259 (1988).

[33] J. McDonald, Phys. Lett. B 225, 133 (1989); S. A. Abel, W. N. Cottingham and I. Whittingham, Phys. Lett. B 244, 327 (1990).

[34] M. U. Rehman, Q. Shafi and J. R. Wickman, Phys. Lett. B 688, 75 (2010) arXiv:0912.4737 [hep-ph]]; J. Ellis, T. E. Gonzalo, J. Harz and W. C. Huang, JCAP 1503, 039 (2015) arXiv:1412.1460 [hep-ph]].

[35] B. Campbell, J. R. Ellis, J. S. Hagelin, D. V. Nanopoulos and K. A. Olive, Phys. Lett. B 200, 483 (1988);

[36] J. R. Ellis, J. S. Hagelin, D. V. Nanopoulos and K. A. Olive, Phys. Lett. B 207, 451 (1988).

[37] B. A. Campbell, J. R. Ellis, J. S. Hagelin, D. V. Nanopoulos and K. A. Olive, Phys. Lett. B 197, 355 (1987).

[38] A. Masiero, D. V. Nanopoulos, K. Tamvakis and T. Yanagida, Phys. Lett. 115B, 380 (1982); B. Grinstein, Nucl. Phys. B 206, 387 (1982).

[39] G. F. Giudice and A. Masiero, Phys. Lett. B 206, 480 (1988).

[40] V. Takhistov [Super-Kamiokande Collaboration], arXiv:1605.03235 [hep-ex]; K. Abe et al. [Super-Kamiokande Collaboration], Phys. Rev. D 90, no. 7, 072005 (2014) arXiv:1408.1195 [hep-ex]].

[41] J. Ellis, J. L. Evans, N. Nagata, D. V. Nanopoulos and K. A. Olive, Eur. Phys. J. C 77, no. 4, 232 (2017) [arXiv:1702.00379 [hep-ph]].

[42] C. Patrignani et al. [Particle Data Group], Chin. Phys. C 40, no. 10, 100001 (2016).

[43] J. R. Ellis, J. L. Lopez and D. V. Nanopoulos, Phys. Lett. B 371, 65 (1996) hep-ph/9510246.

[44] J. R. Ellis, D. V. Nanopoulos and J. Walker, Phys. Lett. B 550, 99 (2002) [hep-ph/0205336. 
[45] J. Hisano, D. Kobayashi and N. Nagata, Phys. Lett. B 716, 406 (2012) arXiv:1204.6274 [hep-ph]].

[46] K. Abe et al. [Super-Kamiokande Collaboration], Phys. Rev. D 95, no. 1, 012004 (2017) arXiv:1610.03597 [hep-ex]].

[47] K. Abe et al., arXiv:1109.3262 [hep-ex].

[48] S. Cecotti, Phys. Lett. B 190 (1987) 86.

[49] A. R. Liddle and S. M. Leach, Phys. Rev. D 68, 103503 (2003) doi:10.1103/PhysRevD.68.103503 [astro-ph/0305263].

[50] J. Ellis, M. A. G. Garcia, D. V. Nanopoulos and K. A. Olive, JCAP 1501, 010 (2015) arXiv:1409.8197 [hep-ph]].

[51] J. R. Ellis and M. K. Gaillard, Phys. Lett. 88B, 315 (1979); D. V. Nanopoulos and M. Srednicki, Phys. Lett. 124B, 37 (1983); C. Panagiotakopoulos and Q. Shafi, Phys. Rev. Lett. 52, 2336 (1984).

[52] H. Georgi and C. Jarlskog, Phys. Lett. 86B, 297 (1979).

[53] P. Minkowski, Phys. Lett. B 67, 421 (1977); T. Yanagida, Conf. Proc. C 7902131, 95 (1979); M. Gell-Mann, P. Ramond and R. Slansky, Conf. Proc. C 790927, 315 (1979) arXiv:1306.4669 [hep-th]]; S. L. Glashow, NATO Sci. Ser. B 59, 687 (1980); R. N. Mohapatra and G. Senjanovic, Phys. Rev. Lett. 44, 912 (1980); R. N. Mohapatra and G. Senjanovic, Phys. Rev. D 23, 165 (1981); J. Schechter and J. W. F. Valle, Phys. Rev. D 22, 2227 (1980); J. Schechter and J. W. F. Valle, Phys. Rev. D 25, 774 (1982).

[54] H. Georgi and D. V. Nanopoulos, Nucl. Phys. B 155, 52 (1979).

[55] M. Fukugita and T. Yanagida, Phys. Lett. B 174, 45 (1986).

[56] G. F. Giudice, A. Notari, M. Raidal, A. Riotto and A. Strumia, Nucl. Phys. B 685 (2004) 89 hep-ph/0310123.

[57] N. Palanque-Delabrouille et al., JCAP 1511, no. 11, 011 (2015) arXiv:1506.05976 [astro-ph.CO]].

[58] I. Esteban, M. C. Gonzalez-Garcia, M. Maltoni, I. Martinez-Soler and T. Schwetz, JHEP 1701, 087 (2017) arXiv:1611.01514 [hep-ph]]; F. Capozzi, E. Di Valentino, E. Lisi, A. Marrone, A. Melchiorri and A. Palazzo, arXiv:1703.04471 [hep-ph].

[59] S. Weinberg, Phys. Rev. Lett. 48, 1303 (1982). 
[60] J. R. Ellis, A. D. Linde and D. V. Nanopoulos, Phys. Lett. B 118, 59 (1982).

[61] D. V. Nanopoulos, K. A. Olive and M. Srednicki, Phys. Lett. B 127, 30 (1983).

[62] J. Ellis, J. Hagelin, D. Nanopoulos, K. Olive and M. Srednicki, Nucl. Phys. B 238 (1984) 453.

[63] M. Y. Khlopov and A. D. Linde, Phys. Lett. B 138, 265 (1984).

[64] J. R. Ellis, J. E. Kim and D. V. Nanopoulos, Phys. Lett. B 145, 181 (1984).

[65] R. Juszkiewicz, J. Silk and A. Stebbins, Phys. Lett. B 158 (1985) 463.

[66] T. Moroi, H. Murayama and M. Yamaguchi, Phys. Lett. B 303, 289 (1993).

[67] M. Kawasaki and T. Moroi, Prog. Theor. Phys. 93, 879 (1995) hep-ph/9403364, hep-ph/9403061].

[68] T. Moroi, hep-ph/9503210.

[69] J. R. Ellis, D. V. Nanopoulos, K. A. Olive and S. J. Rey, Astropart. Phys. 4, 371 (1996) hep-ph/9505438.

[70] G. F. Giudice, A. Riotto and I. Tkachev, JHEP 9911, 036 (1999) hep-ph/9911302.

[71] M. Bolz, A. Brandenburg and W. Buchmuller, Nucl. Phys. B 606, 518 (2001) [Erratumibid. B 790, 336 (2008)] hep-ph/0012052].

[72] K. Kohri, T. Moroi and A. Yotsuyanagi, Phys. Rev. D 73, 123511 (2006) arXiv:hep-ph/0507245.

[73] F. D. Steffen, JCAP 0609, 001 (2006) arXiv:hep-ph/0605306].

[74] J. Pradler and F. D. Steffen, Phys. Rev. D 75, 023509 (2007) hep-ph/0608344.

[75] J. Pradler and F. D. Steffen, Phys. Lett. B 648, 224 (2007) hep-ph/0612291.

[76] V. S. Rychkov and A. Strumia, Phys. Rev. D 75, 075011 (2007) hep-ph/0701104.

[77] M. Kawasaki, K. Kohri, T Moroi and A.Yotsuyanagi, Phys. Rev. D 78, 065011 (2008) arXiv:0804.3745 [hep-ph]].

[78] D. Lindley, Astrophys. J. 294 (1985) 1; J. R. Ellis, D. V. Nanopoulos and S. Sarkar, Nucl. Phys. B 259 (1985) 175; M. Kawasaki and K. Sato, Phys. Lett. B 189 (1987) 23; S. Dimopoulos, R. Esmailzadeh, L. J. Hall and G. D. Starkman, Nucl. Phys. B 311, 699 (1989); J. R. Ellis, G. B. Gelmini, J. L. Lopez, D. V. Nanopoulos and S. Sarkar, 
Nucl. Phys. B 373, 399 (1992); M. Y. Khlopov, Y. .L. Levitan, E. V. Sedelnikov and I. M. Sobol, Phys. Atom. Nucl. 57 (1994) 1393 [Yad. Fiz. 57 (1994) 1466]; E. V. Sedelnikov, S. S. Filippov and M. Y. Khlopov, Phys. Atom. Nucl. 58 (1995) 235 [Yad. Fiz. 58 (1995) 280]; E. Holtmann, M. Kawasaki, K. Kohri and T. Moroi, Phys. Rev. D 60, 023506 (1999) arXiv:hep-ph/9805405; M. Kawasaki, K. Kohri and T. Moroi, Phys. Rev. D 63, 103502 (2001) hep-ph/0012279]; K. Kohri, Phys. Rev. D 64 (2001) 043515 arXiv:astro-ph/0103411]; R. H. Cyburt, J. Ellis, B. D. Fields and K. A. Olive, Phys. Rev. D 67, 103521 (2003) astro-ph/0211258; M. Kawasaki, K. Kohri and T. Moroi, Phys. Lett. B 625 (2005) 7 arXiv:astro-ph/0402490; J. R. Ellis, K. A. Olive and E. Vangioni, Phys. Lett. B 619, 30 (2005) arXiv:astro-ph/0503023; D. G. Cerdeno, K. Y. Choi, K. Jedamzik, L. Roszkowski and R. Ruiz de Austri, JCAP 0606, 005 (2006) arXiv:hep-ph/0509275]; K. Jedamzik, K. Y. Choi, L. Roszkowski and R. Ruiz de Austri, JCAP 0607, 007 (2006) arXiv:hep-ph/0512044; R. H. Cyburt, J. R. Ellis, B. D. Fields, K. A. Olive and V. C. Spanos, JCAP 0611, 014 (2006) astro-ph/0608562; J. Pradler and F. D. Steffen, Phys. Lett. B 666, 181 (2008) [arXiv:0710.2213 [hep-ph]]; S. Bailly, K. Jedamzik and G. Moultaka, Phys. Rev. D 80 (2009) 063509 arXiv:0812.0788 [hepph]]; K. Jedamzik and M. Pospelov, New J. Phys. 11, 105028 (2009) arXiv:0906.2087 [hep-ph]]; M. Pospelov and J. Pradler, Ann. Rev. Nucl. Part. Sci. 60, 539 (2010) arXiv:1011.1054 [hep-ph]]; R. H. Cyburt, J. Ellis, B. D. Fields, F. Luo, K. A. Olive and V. C. Spanos, JCAP 1212, 037 (2012) [arXiv:1209.1347 [astro-ph.CO]].

[79] M. Kawasaki, K. Kohri and T. Moroi, Phys. Rev. D 71 (2005) 083502 arXiv:astro-ph/0408426.

[80] R. H. Cyburt, J. Ellis, B. D. Fields, F. Luo, K. A. Olive and V. C. Spanos, JCAP 0910, 021 (2009) arXiv:0907.5003 [astro-ph.CO]]; R. H. Cyburt, J. Ellis, B. D. Fields, F. Luo, K. A. Olive and V. C. Spanos, JCAP 1010, 032 (2010) arXiv:1007.4173 [astroph.CO]]; R. H. Cyburt, J. Ellis, B. D. Fields, F. Luo, K. A. Olive and V. C. Spanos, JCAP 1305, 014 (2013) arXiv:1303.0574 [astro-ph.CO]].

[81] D. J. H. Chung, E. W. Kolb and A. Riotto, Phys. Rev. D 60, 063504 (1999) hep-ph/9809453.

[82] D. V. Nanopoulos and K. Tamvakis, Phys. Lett. B 110, 449 (1982); M. Srednicki, Nucl. Phys. B 202, 327 (1982); M. Srednicki, Nucl. Phys. B 206, 132 (1982); D. V. Nanopoulos, K. A. Olive and K. Tamvakis, Phys. Lett. B 115, 15 (1982); D. V. Nanopoulos, K. A. Olive, M. Srednicki and K. Tamvakis, Phys. Lett. 124B, 171 (1983).

[83] S. Raby, S. Dimopoulos and L. Susskind, Nucl. Phys. B 169, 373 (1980). 
[84] H. Georgi, L. J. Hall and M. B. Wise, Phys. Lett. 102B, 315 (1981) Erratum: [Phys. Lett. 104B, 499 (1981)].

[85] J. B. Kogut, M. Stone, H. W. Wyld, S. H. Shenker, J. Shigemitsu and D. K. Sinclair, Nucl. Phys. B 225, 326 (1983).

[86] T. Appelquist, K. D. Lane and U. Mahanta, Phys. Rev. Lett. 61 (1988) 1553; D. D. Dietrich and F. Sannino, Phys. Rev. D 75, 085018 (2007) hep-ph/0611341.

[87] R. J. Scherrer and M. S. Turner, Phys. Rev. D 31, 681 (1985).

[88] I. Affleck and M. Dine, Nucl. Phys. B 249, 361 (1985).

[89] R. Easther, R. Galvez, O. Ozsoy and S. Watson, Phys. Rev. D 89, no. 2, 023522 (2014) arXiv:1307.2453 [hep-ph]].

[90] P. Adshead, R. Easther, J. Pritchard and A. Loeb, JCAP 1102, 021 (2011) arXiv:1007.3748 [astro-ph.CO]].

[91] J. Martin and C. Ringeval, Phys. Rev. D 82, 023511 (2010) arXiv:1004.5525 [astroph.CO]].

[92] A.D. Dolgov, and A.D. Linde, Phys. Lett. B116 (1982) 329.

[93] B. A. Campbell, S. Davidson and K. A. Olive, Nucl. Phys. B 399, 111 (1993) hep-ph/9302223.

[94] N. Nagata, K. A. Olive and J. Zheng, JCAP 1702, no. 02, 016 (2017) arXiv:1611.04693 [hep-ph]].

[95] L. Covi, E. Roulet and F. Vissani, Phys. Lett. B 384, 169 (1996) hep-ph/9605319. 